\title{
Bergerac Strains of $C$. elegans Revisited: Expansion of Tc1 elements Impose a Significant Genomic and Fitness Cost
}

\author{
Austin T. Daigle ${ }^{1 \dagger}$, Thaddeus C. Deiss ${ }^{1 \dagger}$, Robert H. Melde ${ }^{1,2}$, Ulfar Bergthorsson $^{1}$, \\ Vaishali Katju ${ }^{1 \dagger \dagger}$ \\ ${ }^{1}$ Department of Veterinary Integrative Biosciences, College of Veterinary Medicine and \\ Biomedical Sciences, Texas A\&M University, College Station, TX 77845, USA \\ ${ }^{2}$ Department of Integrative Biology, University of Wisconsin-Madison, Madison, WI \\ 53706, USA
}

$\dagger$ Joint first authors

†Corresponding author: vkatju@,cvm.tamu.edu

Keywords:

Caenorhabditis elegans | Bergerac | transposable element | transposon | copy-number variation | fitness | whole-genome sequencing | chromatin | recombination

Running Title:

Significant fitness and genomic cost of TE expansion in Bergerac strains of C. elegans 


\section{ABSTRACT}

The DNA transposon Tc 1 was the first transposable element (TE) to be characterized in Caenorhabditis elegans and to date, remains the best-studied TE in Caenorhabditis worms. While Tcl copy-number is regulated at approximately 30 copies in the laboratory $\mathrm{N} 2 /$ Bristol and the vast majority of $C$. elegans strains, the Bergerac strain and its derivatives have experienced a marked Tcl proliferation. Given the historical importance of the Bergerac strain in the development of the $C$. elegans model, we implemented a modern genomic analysis of three Bergerac strains (CB4851, RW6999, and RW7000) in conjunction with multiple phenotypic assays to better elucidate the (i) genomic distribution of $\mathrm{Tc} 1$, and (ii) phenotypic consequences of TE deregulation for the host organism. The median estimates of Tcl copy-number in the Bergerac strains ranged from 451 to 748, which is both (i) greater than previously estimated, and (ii) likely to be an underestimate of the actual copy-numbers since coverage-based estimates and ddPCR results both suggest higher Tcl numbers. All three Bergerac strains had significantly reduced trait means compared to the $\mathrm{N} 2$ control for each of four fitness-related traits, with specific traits displaying significant differences between Bergerac strains. Tcl proliferation was genome-wide, specific to $\mathrm{Tc} l$, and particularly high on chromosomes $\mathrm{V}$ and X. There were fewer Tcl insertions in highly expressed chromatin environments than expected by chance. Furthermore, Tcl integration motifs were also less frequent in exon than non-coding sequences. The source of the proliferation of $\mathrm{Tc} l$ in the Bergerac strains is specific to Tc 1 and independent of other TEs. The Bergerac strains contain none of the alleles that have previously been found to derepress TE activity in C. elegans. However, the Bergerac strains had several Tcl insertions near or within highly germline-transcribed genes which could account for the recent germline proliferation. 


\section{Data Availability}

2 Whole genome sequence files are available in fastq format from the NCBI SRA database

3 under Bioproject PRJNA801693 (https://www.ncbi.nlm.nih.gov/sra/PRJNA801693).

4 Accessions for individual strains sequenced in this study are as follows: CB4851

5 (SAMN25375976), RW6999 (SAMN25375977), RW7000 (SAMN25375978) and Bristol

6 N2 (SAMN25375979). 


\section{INTRODUCTION}

8

Transposable elements (TEs henceforth) are small DNA fragments particularly ubiquitous in eukaryotic genomes that occasionally relocate to new genomic position, hence the moniker of "jumping genes" (McClintock 1948; Chuong et al. 2016). TEs are viewed as parasitic and selfish because they rely on the host genome's cellular machinery to mobilize, and their ability to spread despite deleterious fitness costs associated with disruption of host gene function due to insertions (Orgel and Crick 1980; Muñoz-López and García-Pérez 2010). As such, both prokaryotic and eukaryotic host genomes have ubiquitously evolved complex surveillance and regulatory systems for TE detection and silencing (Sijen and Plasterk 2003; Blumenstiel 2011; Klein and O'Neill 2018; Payer and Burns 2019). Despite their overwhelmingly deleterious effects for host genomes, these mobile genetic elements can also inadvertently contribute to novel advantageous phenotypes as documented in the rapid phenotypic evolution observed in the British peppered moth (van't Hof et al. 2011), the role of TEs in gene transcription as cisregulatory elements in many organisms (Rebollo et al. 2012) and their domestication as a host recombinase mechanism required for genomic rearrangements integral to the adaptive immune systems of higher vertebrate (Yuhang et al. 2019). Recent research has utilized increasingly sophisticated computational tools and a comparative genomics framework to understand the broad genomic effects and functions of TEs (Bourque et al. 2018) while emphasizing the need for studies focusing on the initial proliferation of diverse TE families in diverse taxa (Wells and Feschotte 2020). To fully understand the coevolution between TE regulation and expansion in evolutionary diversification, the mechanisms by which TEs evade regulation at the expense of the host organism requires further elucidation.

The nematode Caenorhabditis elegans has been a useful model for TE research since the discovery of the first C. elegans TE, a DNA transposon named Tcl (Emmons et al. 1983). Tcl and its homologs are among the most abundant TEs occurring in nature (Plasterk et al. 1999). Overall, TEs comprise approximately $12 \%$ of the C. elegans genome (C. elegans Sequencing Consortium 1998). A recent study of TEs in the 
genomes of 208 C. elegans wild isolates revealed significant variation in the copynumber of diverse TE classes (Laricchia et al. 2017), highlighting the contribution of TEs to intraspecific genome variation. Transcription of TEs is under strong purifying selection in C. elegans (Bergthorsson et al. 2020). The primary mechanisms responsible for the regulation of transposons and other foreign DNA in the C. elegans genome are composed of small RNAs (sRNAs), including 21U-RNAs, 26G-RNAs and 22G-RNAs (Ambros et al. 2003), that couple with and guide Argonaute (AGO) proteins (Yigit et al. 2006). Nematode sRNAs have distinct structures and biogenesis pathways, but often share common regulatory functions (Almeida et al. 2019). 21U-RNAs, a special class of sRNA known as PIWI-interacting or piRNAs, were determined to interact directly with the AGO protein prg-1 to prevent TE spread in germ line cells (Batista et al. 2008; Bagijn et al. 2012). Despite evidence that 21U-RNAs play a role in controlling TE transcription, only the mariner transposon $T c 3$ has been demonstrated to become mobilized upon disruption of piRNA machinery (Das et al. 2008; Reed et al. 2020). High-throughput sequencing of piRNAs and siRNAs in various mutants seemed to suggest that WAGOclass 22G-RNAs played a larger role in transposon silencing, and it had been suggested that the mutants studied could have inherited long-term effects of piRNA silencing due to multigenerational epigenetic memories (Ashe et al. 2012; Reed et al. 2020). However, a recent experimental study in C. elegans demonstrated that 22G-RNA epimutations are typically unstable with an average persistence time of two to three generations (Beltran et al. 2020). Many details and overlapping features of the intricate regulatory pathways involved in silencing TEs and other germline transcripts in C. elegans await further elucidation.

The Bergerac strain of $C$. elegans is of historical importance in the development of the species as a model organism (Riddle et al. 1997). Bergerac was one of the earliest laboratory strains of C. elegans to be cultured and was isolated from garden soil in 1944 in Bergerac, France by Victor Nigon of the Université de Lyon (Nigon 1949; Nigon and Félix 2017). Some of the earliest genetic work in C. elegans occurred with the use of the Bergerac strain, including the discovery of a Mendelian temperature-sensitive allele (Fatt and Dougherty 1963). Owing to difficulties with propagation later determined to be due 
to temperature-sensitivity of hermaphrodites (Fatt and Dougherty 1963), infertile males (Fatt and Dougherty 1963), and high mutation rates (Moerman and Waterston 1984), the use of the Bergerac strain for subsequent genetic work was abandoned and replaced by the Bristol N2 strain isolated by L. N. Staniland from a mushroom compost heap in Bristol, England (Nicholas et al. 1959; Brenner 1974; Riddle et al. 1997; Nigon and Félix 2017). Several decades later and with a more developed arsenal of molecular and genetic tools available, several $C$. elegans researchers took to investigating the mechanistic bases of the aberrant phenotypes associated with the Bergerac strain. Bristol N2 and Bergerac were observed to exhibit differences in restriction endonuclease cleavage patterns on Southern hybridizations when probed with randomly selected cloned fragments (Emmons et al. 1979, 1983; Files et al. 1983). Southern hybridization analyses further demonstrated that while Bristol N2 possessed $20 \pm 5$ dispersed copies of Tcl in the genome, the Bergerac strain appeared to have an estimated $200 \pm 50$ copies (Emmons et al. 1983). Further investigation of Tc 1 copy-number in ten newly available C. elegans isolates led Emmons et al. (1983) to the parsimonious conclusion that these intraspecific differences in transposon copy-number were owing to the uniquely massive proliferation of Tcl elements in the Bergerac strain which likely resulted in gene disruption leading to phenotypic defects. A later study using quantitative dot blot hybridization presented estimates of Tcl copy-number in the Bristol N2 and Bergerac strain as being $\sim 30 \mathrm{Tc} 1$ and 300-550 copies, respectively (Egilmez et al. 1995). Rosenzweig et al. (1983a) were the first to generate the complete $1,610 \mathrm{bp}$ nucleotide sequence of the Tc 1 transposable element in C. elegans with its characteristic short inverted terminal repeats of $<100 \mathrm{bp}$. Liao et al. (1983) provided a substantially detailed characterization of this C. elegans Tcl, namely that (i) while it shared many structural features with other eukaryotic DNA transposable elements, it belonged to a unique class, and (ii) all C. elegans Tc 1 copies appeared to display full-length conservation unlike Drosophila P-elements, suggesting that it encodes products mediating its own transcription. Tcl elements in the Bergerac strain were found to be significantly more active relative to their counterparts in the Bristol N2 genome, displaying site-specific insertion and excision from the muscle gene unc-54 (Eide and Anderson 1985, 1988). In addition to increased transposition, it was noted that Bergerac strains were less fit, produced fewer progeny, moved with less 
100 coordination, and had a higher incidence of males relative to other C. elegans strains,

101 despite the males being sterile (Hodgkin and Doniach 1997). It has been hypothesized

102 that the increase in Tcl copy-number occurred in the laboratory after Nigon isolated

103 Bergerac from the wild (Moerman and Waterston 1984; Egilmez et al. 1995; Nigon and

104 Félix 2017). However, the cause of the massive proliferation of Tcl elements in the

105 Bergerac strains remains to be identified.

106

While some traits in Bergerac have been studied, mainly in the strain RW7000 (Fatt and Dougherty 1963; Shook and Johnson 1999; Vertino et al. 2011; Lee et al. 2016), a comparative study of multiple fitness-related traits and precise quantification of composite fitness has yet to be conducted on multiple distinct Bergerac strains simultaneously. This project is the first to employ high-throughput Illumina wholegenome sequencing technology to sequence and analyze the entire genomes of three distinct Bergerac strains. While the genome of one Bergerac strain, CB4851, has previously been sequenced, it has not yet been analyzed to study $\mathrm{Tc} 1$ proliferation (Cook et al. 2016; Laricchia et al. 2017). Herein, we quantified and compared four fitnessrelated traits (developmental rate, productivity, longevity, and survivorship) in order to discern extant phenotypic variation among the Bergerac strains RW6999, RW7000, and CB4851. Next, using whole-genome sequencing and ddPCR data for each strain, we generated more accurate TE copy-number estimates, quantify the decreased and variable fitness across Bergerac strains relative to Bristol N2, analyze the distribution and sequence context of Tcl landing sites, and conduct an initial search for the cause of increased Tc 1 proliferation.

\section{MATERIALS AND METHODS}

Bergerac strains of C. elegans used in this study

This study focused on three subclones of the original Bergerac strain isolated by Nigon in Bergerac, France in 1944 (Nigon and Félix 2017). This strain was shared with several other laboratories before the implementation of cryopreservation techniques for 
131 C. elegans, and hence the names of these subclones of the original Bergerac strain either

132 represent their initial culture location or standardized strain nomenclature that came to be

133 adapted later. We focused on three Bergerac strains to quantify their fitness declines and

134 genomic divergence that may have occurred during laboratory evolution and divergence

135 following the proliferation of Tcl elements. The first, RW7000 (also known as Bergerac

136 BO for its location in Boulder, Colorado, USA), belongs to the Boulder BO sublineage

137 and was given to David Hirsh by Nigon's student Jean-Louis Brun in 1983, and used in

138 many of the original studies of TEs in C. elegans (Liao et al. 1983; Rosenzweig et al.

139 1983a; Moerman and Waterston 1984; Mori et al. 1988; Nigon and Felix 2017). A

140 second Bergerac strain, RW6999, is relatively understudied and reported to be a subclone

141 of RW7000 as per the Caenorhabditis Genetics Center (CGC)

142 (https://cgc.umn.edu/strain/RW6999). A third strain, CB4851, belonging to the

143 Cambridge sublineage (Nigon and Felix 2017), was shared with Sydney Brenner in 1969

144 (Hodgkin and Doniach 1997; Nigon and Félix 2017).

Fitness assays for four life-history traits and statistical analyses of fitness data

To quantify the fitness of Bergerac strains RW7000, RW6999, and CB4851

relative to the laboratory strain Bristol N2 (also referred to as N2), we assayed four fitness-related, life-history traits, namely (i) productivity, (ii) survivorship to adulthood

151 (also referred to as survivorship), (iii) longevity, and (iv) developmental rate as previously described (Katju et al. 2015, 2018; Dubie et al. 2020). All assays were conducted on Nematode Growth Medium (NGM) agar plates seeded with the E. coli

154 strain $\mathrm{OP} 50$ at $20^{\circ} \mathrm{C}$, the standard temperature for C. elegans culturing. Cryopreserved 155 stocks of RW7000, RW6999, and CB4851 and N2 were thawed and individual worms 156 isolated onto NGM plates. In order to establish independent lines, 15 and 20 worms were 157 isolated for the wild type N2 and each Bergerac strain, respectively. After the 158 establishment of multiple lines per strain, the worms were allowed to self and five of their 159 L4 larval stage progeny were isolated singly onto a new plate, thereby establishing five 160 sublines per line (total $15 \times 5=75$ for N2; $20 \times 5=100$ for each Bergerac strain). To 161 negate the possibility of maternal or grandmaternal effects (Lynch 1985), each replicate 
162 was propagated by single-worm transfer for one more generation. This hierarchical

163 structure (strains, lines, and sublines) combined with the fact that hermaphrodites self-

164 fertilize to produce the next generation, minimizes genetic divergence among replicates,

165 and allows for a clean estimate of environmental variance by comparison of sublines.

166 Three fitness assays (development, productivity, and longevity) were conducted on a

167 single, third-generation worm isolated from each subline as an L1 larva. To measure the

168 developmental rate, commencing $36 \mathrm{~h}$ after the initial isolation of the L1 larva, each worm

169 was checked every $2 \mathrm{~h}$ to identify the time (in hours) until the first egg was visible in the

170 worm's uterus. Upon noting the presence of the first egg in the uterus, the worm was

171 scored as having developed to adulthood. This initial measurement of the time (hours)

172 from L1 larva to an egg-bearing adult yielded the developmental time. The inverse of the

173 developmental time yielded the developmental rate. Worms that died before reaching

174 adulthood were not scored.

175

176

177

178

179

180

181

182

183

184

185

186

187

188

189

190

191

To assay productivity, each worm that developed to adulthood was transferred to a new plate every $24 \mathrm{~h}$ for eight consecutive days. After transferring the worm to a new plate, the eggs from the previous plate were allowed to hatch for an additional $24 \mathrm{~h}$, then stored at $4^{\circ} \mathrm{C}$ for a minimum of one month to allow the progeny to die without producing offspring. Counts were conducted by staining each plate with a $0.075 \%$ water dilution of toluidine blue, which temporarily makes the progeny stand out white against a purple background to facilitate visualization for worm counts.

Following eight days of daily transfers to a fresh plate as part of the productivity assay, each worm was transferred to a fresh NGM agar plate seeded with the E. coli OP50 until death in order to score longevity in days. In order to score longevity, the worms were monitored each day for movement and pharyngeal pumping. When no movement was detected, the agar pad near the worm was gently tapped. If no response was detected, the tail of the worm was tapped. If the worm failed to respond, it was recorded as dead and the days to mortality were noted. 
To assay survivorship to adulthood, we used 10 L1 siblings of each thirdgeneration worm assayed for the preceding three life-history traits. The $10 \mathrm{~L} 1$ larvae were isolated onto a seeded 60mm NGM agar plate. For some sublines, less than $10 \mathrm{~L} 1$ individuals were isolated due to the low and delayed productivity of Bergerac worms. $36 \mathrm{~h}$ after isolation, the plates were checked for worms that survived to adulthood, and each plate was scored using the fraction of worms that survived to adulthood (values ranged from 0 to 1 ). For plates with desiccated worms on the edge of the plate, worms were still scored as surviving if eggs were observed in the uterus, to provide a more conservative estimate of survivorship.

For each of the four fitness traits, a two-level nested Model I ANOVA with unequal sample sizes (Sokal and Rohlf 1995) was employed to partition the total phenotypic variance into among- and within line-components. The highest level of classification tested for a treatment effect (four strains as treatments: (i) wild type control N2, (ii) strain RW7000, (iii) strain RW6999, and (iv) strain CB4851. The next level of hierarchy tested for a subgroup variance component (difference among independent lines within a treatment). The last hierarchical level estimated the subline variance. To conduct pairwise comparisons for all strain pairs per fitness trait, a Tukey-Kramer HSD test for unequal sample sizes was used at a 5\% experiment-wise error rate.

\section{Motility Assays}

In addition to the fitness assays, motility assays were conducted to quantify several locomotory traits in the Bergerac strains relative to the laboratory strain N2. Cryopreserved stocks of each strain were thawed and bottlenecked for three generations to establish five replicates for each strain, using procedures similar to the previously described fitness assays. Two identical assays were conducted on separate days, resulting in recordings of 10 plates for each strain in total. When the progeny of each thirdgeneration replicate reached the L4 life stage, 10 hermaphrodite siblings were isolated onto a single $35 \times 100 \mathrm{~mm}$ plate with $3.25 \mathrm{~mL}$ NGM. 21-22h after isolating L4s for each strain, the young adult worms were removed from a $20^{\circ} \mathrm{C}$ incubator and transferred to an 
unseeded NGM plate. Within 3 min of the transfer, a 60s video was recorded using Zen on an Axio Zoom.V16 Zoom microscope equipped with an AxioCam 503 Color camera at $5 \mathrm{fps}$. The objective lens used for this analysis was the Zeiss PlanNeoFluar Z 1x/0.25 FWD 56mm. The conversion factor for videos provided by the Zen program was 25.94 $\mu \mathrm{M} /$ pixel. The resolution of the final videos was $968 \times 730$. The order we recorded strains was randomized, and worms were kept in the incubator as long as possible to limit environmental variance. The temperature in the recording room was recorded at least three times for each assay and remained $\sim 21 \pm 1^{\circ} \mathrm{C}$. These details are provided to increase the reproducibility of our motility assay in a manner consistent with the suggestions of Angstman et al. (2016).

A custom Matlab program called Zentracker was used to analyze assay videos (https://github.com/wormtracker/zentracker). After inputting the scaling factor provided by the scope, all videos were tracked at intensities ranging from 130-180, then manually validated to ensure all worms were tracked and non-worm objects were removed. After checking the validity, the values for average speed, length, area and direction change were extracted for each video. In this analysis, averages were defined as the overall average value of all the valid datapoints.

\section{DNA extraction and Illumina sequencing}

Genomic DNA was extracted from Bergerac and N2 control lines as previously described (Konrad et al. 2018) with libraries prepared using the Nextera DNAflex library kit (Illumina, San Diego, CA). Libraries were sequenced on the Illumina Novaseq6000 platform $(2 \times 150 \mathrm{bp})$ at the North Texas Genome Center at the University of Texas at Arlington.

\section{Tc1 copy-number estimation with ddPCR}

Digital droplet polymerase chain reaction (ddPCR) copy-number variation (CNV) assay was performed following the Bio-Rad ddPCR Copy-Number Variation Assay 
protocol (https://www.bio-rad.com/webroot/web/pdf/lsr/literature/10033173.pdf) for lines N2, CB4851, RW6999, and RW7000. The ddPCR utilizing a Tc1 targeting probe and a daf-3 (a single copy reference) targeting probe with Fluorescein (FAM) were analyzed on the Bio-Rad QX ONE ddPCR system. The haploid genome-wide copy-number of Tcl was determined based on the estimated copy-number of Tcl-positive relative to the $d a f-3$ single-copy control. The reactions were run using two concentrations of template genomic DNA: (i) the first, at $0.1 \mathrm{ng} / \mu \mathrm{L}$, to allow a sufficient number of $d a f-3$ positive droplets, and (ii) another, a 100 -fold dilution of $0.001 \mathrm{ng} / \mu \mathrm{L}$, to avoid saturation of positive Tcl droplets. The number of $d a f-3$ positives was then divided by the dilution factor to identify the proportion of negative droplets for Poisson calculations shown below. The cutoffs for positive and negative droplets were assigned manually on the QX Manager Software (1.2 Standard Edition) provided by Bio-Rad based on the separation of the droplets.

\section{Tc1 copy-number estimation from whole-genome sequence (WGS) data}

Tc 1 copy-number in each genome was also estimated using the McClintock metapipeline, v2.0.0 (Nelson et al. 2017) which combines many TE-detection algorithms to identify reference and nonreference TE insertions in each genome. A standard consensus sequence of a 1,610 bp Tcl element was used as an input. Each TE-detection algorithm produces unique results containing both correct calls and false positives, with varying degrees of sensitivity and precision in simulated and real datasets (Nelson et al. 2017; Vendrell-Mir et al. 2019). McClintock v2.0.0 provides an estimate of normalized mean coverage. Five callers were chosen from the McClintock pipeline to estimate Tcl copynumber based on the absolute difference from the normalized mean coverage and ddPCR copy-number estimate: ngs_te_mapper2 (Han et al. 2021), RelocaTE (Robb et al. 2013), TEMP2 (Yu et al. 2021), RetroSeq (Keane et al. 2013), and TEFLoN (Adrion et al. 2017).

To visualize the overlap between the results of the various McClintock callers, the reference genome (PRJNA13758.WS279) was split into 1,000 bp windows using 
285 BEDTools (Quinlan and Hall 2010). The BEDTools "intersect" command was used to

286 create a file with a window for each non-reference Tc 1 call from each McClintock caller.

287 Given that the reference Tc 1 elements tend to span several 1,000 bp windows, a similar

288 procedure was used to assign reference calls to $100,000 \mathrm{bp}$ windows, and subsequently

289 these files were combined with the nonreference windows. Using a web Venn diagram

290 tool (http://bioinformatics.psb.ugent.be/webtools/Venn/), Venn diagrams describing the

291 overlap of Tcl calls for each method in each strain were produced, along with a Venn

292 diagram describing the overlap of TEMP2 Tc1 calls between the Bergerac strains. copy-number for other TEs, namely $T c 2, T c 3, T c 4 v, T c 5, T c 6, T c 9, T U R M O I L 1$,

Tc1 landing site sequence analysis

Due to the high positional accuracy of the TEMP2 caller $(\sim 0.90)$ in the prediction of synthetic TE insertions used as benchmarks in the original McClintock publication, it was chosen for analyses of TE landing sites (Nelson et al. 2017). The sequence context of each Tc 1 insertion predicted by TEMP 2 was analyzed in each Bergerac strain. The FASTQ reads generated by Illumina whole-genome sequencing were aligned to the $C$. elegans reference genome (PRJNA13758.WS279) with the Burrows-Wheeler Aligner (BWA), version 0.7.12-r1039. Using the BED file generated by the McClintock pipeline, sequences $\pm 25 \mathrm{bp}$ from each insertion site were extracted from the BWA alignments. These sequences were aligned using ClustalW and a consensus sequence was generated for each strain with IUPAC nucleotide code by analyzing the distribution of nucleotides \pm

Statistical analyses of TE distribution in the Bergerac genomes

To assess the environment of Tc 1 landing sites within the Bergerac strains' genomes, genomic coordinates specific to various genomic features were used to define 
316 the genomic context of Tcl insertions. Annotation files were created for chromosomal

317 arms, cores, and tips, exon, intron, or intergenic regions, as well as various histone

318 modification environments. To preclude overlapping regions with the annotation files

319 from yielding multiple counts, these annotation files were then merged to prevent

320 overlapping regions within the annotation files from yielding multiple counts using the

321 "merge" command of the BEDTools software package (Quinlan and Hall 2010). Finally,

322 the merged BED files denoting the landing site environment and the resulting Tcl BED

323 files for each line were analyzed for overlap using the bedtools "intersect" command. For

324 exon and intron overlap with identified Tc 1 sites, the merged exon and intron annotations

325 were analyzed for intersections with the "-v" flag ensuring only intron exclusive genomic

326 sites used to call intronic sites. The resulting counts were used as input for chi-squared

327 tests, using the proportion of genomic coverage to calculate expected values within the $\mathrm{R}$

328 statistical analysis software (R Core Team 2014).

Genes with exons disrupted by Tc1

Using the $C$. elegans N2 reference annotations file from WormBase (version WS279; www.wormbase.org), a table of genes with predicted exonic or intronic Tc1 insertions according to TEMP2 was generated along with a list of intergenic insertions.

337 (https://wormbase.org/tools/mine/simplemine.cgi), a table containing a description of

338 RNAi phenotypes, allele phenotypes, concise descriptions, and automated descriptions

339 for genes disrupted by Tc 1 was created. 
JU394, NIC1049, and NIC2. One isolate, ECA243, is a renamed sample of the strain CB4851 that was previously sequenced. The BAM alignments for the seven chosen natural isolates were obtained from the CeNDR database, release 20210121 (Cook et al. 2017). The reads for the Bergerac strains and our laboratory sample of N2 were cleaned with Fastp using default settings and aligned with BWA-MEM (Burrows-Wheeler Aligner maximal exact match) to the $C$. elegans reference genome PRJNA13758.WS276 to replicate the CeNDR procedure. All 11 BAM files were then merged, and MiModd was used to extract SNPs and indels for all strains. These variants were filtered to keep homozygous variants with a minimum depth of 3. SNPeff, a tool to predict the effect of SNPs on protein-coding genes, was used to annotate these variants (Cingolani et al. 2012). In addition to SNPeff, SIFT $4 \mathrm{G}$ was used to predict which amino acid changes in the Bergerac strains are likely to be deleterious to protein function. Deletions were also called for all strains using MiModd, with a maximum coverage of 4 and a minimum size of $100 \mathrm{bp}$. After calling variants and deletions, the mutations were filtered to retain homozygous variants identified in all three Bergerac strains and absent in all nonBergerac strains. Genes with deletions were determined using the C. elegans WS276 reference gff3 file. Summary tables for all mutated genes were created using SimpleMine. Finally, to place the Bergerac strains in a phylogenetic context, we ran a maximum likelihood analysis with PhyML (Guindon et al. 2010), using a GTR substitution model with a discrete gamma distribution and four rate categories on $11 C$. elegans strains, which included the three Bergerac strains and N2.

\section{RESULTS}

Significant variation in Tcl copy-number among the Bergerac strains

The standard laboratory strain of C. elegans, Bristol N2, has diverged genetically since it was first isolated through the independent accumulation of base substitutions, copy-number changes and TE activity during propagation in different laboratories (Lipinski et al. 2011). The estimated number of Tcl elements in low Tcl copy-number strains, such as Bristol N2 typically ranges from 20-30 copies (Emmons et al. 1983; Liao 
et al. 1983; Egilmez et al. 1995). The median and average number of Tcl elements in our reference Bristol N2 was 28, with a range of 26-30 copies based on five methods (ngs_te_mapper 2, RelocaTE, TEMP2, Teflon, Normalized TE coverage) implemented in the McClintock pipeline (Figure 1A, Table 1, Supplemental Table S1). The RetroSeq caller of the McClintock program does not call non-reference TEs; hence an estimate was omitted for the N2 strain. ddPCR results from our N2 control estimated the Tc1 copynumber as 29 .

The three Bergerac strains display substantial variation in Tc1 copy-number that is consistent between different variant callers notwithstanding some variation in results between different methods (Figure 1A, Table 1). The range in Tcl copy-number estimates among the Bergerac strains was non-overlapping (Table 1). We first estimated Tcl copy-number in the three Bergerac strains by employing six computational methods/callers available in the McClintock pipeline (ngs_te_mapper 2, RelocaTE, RetroSeq, TEMP2, TEFLoN, and Normalized TE coverage). CB4851 harbored the lowest number of Tc 1 elements with a mean and median of 465 and 451, respectively. The mean and median of Tcl copy-number in RW6999 was 593 and 581, respectively. RW7000 has the highest abundance of Tc 1 elements with a mean and median estimate of 759 and 748, respectively (Figure 1B, Table 1). The estimates based on normalized Tc1 coverage were $12-21 \%$ greater than those based on the five methods that use paired-ends and split reads to estimate copy-number. BED files containing the locations of all calls produced by McClintock are provided in Supplemental Table S1. ddPCR estimates of Tcl copy-number in the three Bergerac strains were consistently higher than their corresponding median copy-number estimates from McClintock, ranging from 495, 610, and 882 copies for CB4851, RW6999 and RW7000, respectively. Both ddPCR and McClintock estimates support the general conclusion that the three Bergerac strains can vary considerably with respect to Tcl copy-number.

To assess whether McClintock component methods were calling Tcl insertions in similar locations, Tcl calls produced by ngs_te_mapper2, RelocaTE, TEMP2, RetroSeq, and TEFLoN were assigned to 1,000 bp windows and compared (Figure 2). The vast 
majority of Tc 1 calls are supported by multiple callers, with $70 \%$ of the 523 unique $\mathrm{Tc} l$ calls in CB4851 supported by all five callers, and $83 \%$ of calls supported by four or more callers. In addition to the comparison of Tcl insert locations between callers, the overlap of insert locations between the Bergerac strains was assessed by comparing the locations of calls produced by TEMP2 (Supplemental Figure S1). Though a fraction of insert sites are shared between all three strains, the Bergerac strains display high variability in the location of insert sites, with large differences observed between the strain CB4851 and the two RW strains. This pattern reflects continued Tc 1 mobility and proliferation during the separate laboratory cultivations of these strains reported by Nigon and Felix (2017), with the RW strains sharing a more recent common ancestor.

\section{Variation in non-Tcl elements in the Bergerac strains}

The McClintock package was also used to estimate the copy-number of a wider variety of TEs in the three Bergerac strain relative to N2 (Supplemental Table S1).

There were no differences in the estimated counts between N2 and the Bergerac strains for the majority of non-Tc1 elements (Mariner2-5, Tc3, Tc6, Tc9, Turmoil1 and 2)

(Figure 1B). However, the Bergerac strains appear to have more Tc2 elements than N2. The average estimated count of Tc2 in was three and 20 in N2 and the Bergerac strains, respectively. Coverage estimates using normalized read depth ranged between 25-30 in the Bergerac strains and four in N2. The average counts of Tc4 and Tc5 appeared to be higher in CB4851 than in the other two Bergerac strains (RW6999 and RW7000) and N2. However, the results were highly variable between different callers in McClintock, especially for Tc5 where the number of counts ranged from $0-40$, and the distribution of counts exhibited considerable overlap between the four strains. Furthermore, normalized read depth did not provide support for differences in Tc5 counts between the strains, with estimates of 13-14 elements per strain.

\section{Bergerac strains have significantly lower fitness than the wild type N2 strain}


Given that the Bergerac strain was one of the earliest $C$. elegans isolates to be cultured under laboratory conditions (Nigon and Felix 2017), preceding studies have described its temperature-sensitivity (Fatt and Dogherty 1963), low fertility (Abdulkader and Brun 1980; Lee et al. 2016), and other phenotypic abnormalities (Hodgkin and Doniach 1997). This study aimed to empirically quantify the fitness of the three Bergerac strains (CB4851, RW6999, RW7000) relative to the Bristol N2 strain by measuring four fitness traits, namely productivity, survivorship to adulthood, longevity, and developmental time. The phenotypic fitness assays comprised measurements on five replicates each, where possible, of 15 N2 lines and 20 lines each of the three Bergerac strains. The mean fitness values for each measured trait are displayed in Figures 3A-D and Table 2.

The mean productivity of the N2, CB4851, RW6999, and RW7000 strains was 309, 75, 107, and 43 offspring, respectively (Figure 3A; Table 2). The three Bergerac strains exhibited a $65-86 \%$ reduction in mean productivity relative to the $\mathrm{N} 2$ strain (Figure 3E). As reported in a previous study of Bergerac-derived recombinant inbred lines (RILs) (Shook and Johnson 1999), many Bergerac worms were observed to die by matricidal hatching ("worm bagging" or "bagging"), a phenotype characterized by larvae hatching within the hermaphrodite before egg-laying occurs. However, these worms were observed to lay some eggs before bagging and were included in the analysis to provide a realistic estimate of mean productivity in laboratory conditions. ANOVA analyses found a significant variance component for productivity among the four strains $\left(F_{S}{ }^{\prime}=309.93 ; p\right.$ $<0.0001$ ) whereas among-line divergence was nonsignificant (Table 3).

The mean survivorship to adulthood of the N2, CB4851, RW6999, and RW7000 strains was 98, 91, 89, and 83\%, respectively (Figure 3B; Table 2). The three Bergerac strains exhibited a $7-15 \%$ reduction in mean survivorship relative to the N2 strain (Figure 3E). ANOVA analyses found a significant variance component for survivorship among the four strains $\left(F_{s}^{\prime}=16.8 ; p<0.0001\right)$ as well as among-line divergence $\left(F_{s}=\right.$ 1.42; $p<0.05$ ) (Table 3). As was observed for productivity, RW7000 exhibited the lowest survivorship among the three Bergerac strains relative to N2. 
The mean longevity of the N2, CB4851, RW6999, and RW7000 strains was 14, 10, 9, and 10 days, respectively (Figure 3C; Table 2). The three Bergerac strains exhibited a $29-36 \%$ reduction in longevity relative to the N2 strain (Figure 3E). ANOVA analyses found a significant variance component for longevity among the four strains $\left(F_{s}^{\prime}\right.$ $=12.49 ; p<0.0001$ ) whereas among-line divergence was nonsignificant (Table 3).

The mean developmental time of the N2, CB4851, RW6999, and RW7000 strains was 48, 60, 53, and 60 hours, respectively (Figure 3D; Table 2). Hence, all three Bergerac strains exhibited delayed development to reproductive maturity relative to $\mathrm{N} 2$ (10-25\% longer). ANOVA analyses found a significant variance component for developmental time among the four strains $\left(F_{s}{ }^{\prime}=34.06 ; p<0.0001\right)$ whereas among-line divergence was nonsignificant (Table 3). We further calculated the developmental rate as the inverse of the relative developmental time. Relative to N2, the three Bergerac strains exhibited a 9-20\% reduction in their developmental rate (Figure 3E), with RW6999 and RW7000 exhibiting the greatest reduction in developmental rate.

The fitness assays additionally detected significant differences among Bergerac strains for all fitness-related traits. We conducted comparisons among pairs of means based on unequal sample sizes using the Tukey-Kramer HSD method to determine which strain pairs differed significantly from each other for the four assay fitness traits (Figures 3A-E, Supplemental Tables S2-S5). All three Bergerac strains were significantly different from N2 for each of the four fitness traits. With respect to productivity, all pairwise strain comparisons were significant with the relationship expressed as follows: RW7000 > CB4851 > RW6999 > N2 (Table 2, Supplemental Table S2). With the exception of the CB4851 vs. RW6999 comparison, all other pair-wise strains comparisons showed significant differences for survivorship to adulthood (RW7000 <

498 Supplemental Table S3). Survivorship to adulthood of the four strains showed the 499 following trend: RW7000 > RW6999/CB4851 > N2. While all of the three Bergerac 500 strains had significantly reduced mean longevity relative to $\mathrm{N} 2$, they did not differ 
501 significantly from each other with all Bergerac strains surviving an average of 9-10 days

502 after the L1 larval stage N2 (Table 2, Supplemental Table S4). With the exception of

503 the CB4851 vs. RW7000 comparison, all other pair-wise strains comparisons showed

504 significant differences for mean developmental time (RW6999 < RW7000, $p=1.87 \times 10^{-}$

505 6; RW6999 < CB4851, $p=5.94 \times 10^{-5}$ ). (Table 2, Supplemental Table S5).

506 Developmental time of the four strains showed the following trend: N2 > RW6999

$507>$ RW7000 > CB4851/RW7000.

Phenotypic divergence of Bergerac strains with respect to motility- and size-related traits

The motility assays revealed variable levels of phenotypic divergence for several

513 (speed, direction, body length and body area) in the wild type N2 strain. Our analysis of

514 the average speed and direction change of N2 worms was concordant with the values

515 observed by Angstman et al. (2006), despite our use of a different worm tracking

516 program. Our N2 average speed was $177.8 \mu \mathrm{m} / s$ with an SD of $48.9 \mu \mathrm{m} / \mathrm{s}(\mathrm{SE} 15.5)$

517 compared to an average $146.9 \mu \mathrm{m} / \mathrm{s}$ with an SD of $55.3 \mu \mathrm{m} / \mathrm{s}$ measured by Angstman et

518 al. (2006). Similarly, our N2 average direction change was 0.531 radians $/ s$ with an SD of

5190.218 radians/s (SE 0.069) compared to Angstman et al.'s (2006) average direction

520 change of $0.70 \mathrm{radians} / \mathrm{s}$ with an $\mathrm{SD}$ of $0.30 \mathrm{radians} / \mathrm{s}$, after converting their

521 measurements from degrees to radians. Similarly, the average length of our N2 worms

$522(799 \mu \mathrm{m}, \mathrm{SD} 56.2 \mu \mathrm{m}, \mathrm{SE} 17.8)$ is close to a previously reported length of $\mathrm{N} 2$ after the

523 fourth larval molt, $850 \mu \mathrm{m}$ (Byerly et al. 1976). Trait means for speed, body length, body

524 area, and direction change are reported in Figures 4A-D and Table 4. As each data point

525 represents the average of ten worms on a plate for 1min, a one-level ANOVA test was

526 used to reveal significant differences for each trait measured (speed, direction, body

527 length, and body area). We additionally conducted comparisons among pairs of means

528 using the Tukey-Kramer HSD method to determine which strain pairs differed

529 significantly from each other for the four motility- and size-associated traits (Figures 4A-

530 E, Supplemental Tables S6-S9). 
The mean speed of the N2, CB4851, RW6999, and RW7000 strains was 178, 166, 121, and $53 \mu \mathrm{m} / \mathrm{s}$, respectively (Figure 4A; Table 4). The three Bergerac strains exhibited $\sim 7-70 \%$ reduction in speed relative to the N2 strain (Figure 4E). ANOVA analyses found a significant variance component for speed among the four strains $\left(F_{s}=\right.$ 37.57; $p=3.50 \times 10^{-11}$ ) (Table 5). With the exception of the CB4851 vs. N2 comparison, all other pair-wise strains comparisons showed significant differences for mean speed $\left(\mathrm{RW7000}<\mathrm{CB} 4851, p=1.51 \times 10^{-9}\right.$; RW7000 < RW6999, $p=4.14 \times 10^{-5}$; RW6999 < CB4851, $\left.p=8.30 \times 10^{-3}\right)($ Table 4, Supplemental Table S6). Strain speed showed the following trend: N2/CB4851 > RW6999 > RW7000.

The mean body length of the N2, CB4851, RW6999, and RW7000 strains was 799, 647, 675, and $636 \mu \mathrm{m}$, respectively (Figure 4B; Table 4). The three Bergerac strains exhibited $\sim 16-20 \%$ reduction in body length relative to the N2 strain (Figure 4E). ANOVA analyses found a significant variance component for body length among the four strains $\left(F_{s}=22.58 ; p=2.14 \times 10^{-8}\right)($ Table 5). While all of the three Bergerac strains had significantly reduced mean body length relative to N2, they did not differ significantly from each other (Table 4, Supplemental Table S7). Hence, body length showed the following trend: N2 > CB4851/RW6999/RW7000.

The mean body area of the N2, CB4851, RW6999, and RW7000 strains was 61078, 50758, 49966, and $42266 \mu \mathrm{m}^{2}$, respectively (Figure 4C; Table 4). The three Bergerac strains exhibited $\sim 17-31 \%$ reduction in body area relative to the N2 strain (Figure 4E). ANOVA analyses found a significant variance component for body area among the four strains $\left(F_{s}=19.59 ; p=1.06 \times 10^{-7}\right)($ Table 5). All three Bergerac strains had significantly reduced mean body area relative to N2 (Supplemental Table S8). The three Bergerac strains also exhibited some significant differences amongst each other with respect to body area. While the CB4851/RW6999 pair comparison was nonsignificant, RW7000 had significantly smaller body area relative to both CB4851 ( $p=$ $\left.7.73 \times 10^{-3}\right)$ and RW6999 $\left(p=1.78 \times 10^{-2}\right)($ Supplemental Table S8). Body area showed the following trend: N2 > CB4851/RW6999 > RW7000. Because all worms in these motility assays were approximately the same age, these size differences could be 
563 explained by the previously observed variation in developmental time. A Pearson test

564 between the average developmental time for each strain and body area confirms that

565 these variables are correlated $\left(p=1.17 \times 10^{-6}\right)$.

566

567

568

569

570

571

572

573

574

575

576

577

578

579

580

581

582

583

584

585

586

587

588

589

590

591

592

593

The mean direction change of the N2, CB4851, RW6999, and RW7000 strains was $0.53,0.63,0.59$, and 1.13 radians $/ s$, respectively (Figure 4D; Table 4). The three Bergerac strains exhibited $11-113 \%$ increase in direction change relative to the N2 strain (Figure 4E). ANOVA analyses found a significant variance component for direction change among the four strains $\left(F_{s}=28.15 ; p=p=1.50 \times 10^{-9}\right)$ (Table 5). However, only RW7000 was significantly different from the three other strains (N2, and the other two Bergerac strains) with respect to direction change N2 (Supplemental Table S9). The average direction change of strain RW7000 was much larger than the other Bergerac strains (RW6999 < RW7000, $p=6.70 \times 10^{-8}$; CB4851 $<\mathrm{RW7000,p=3.72 \times}$ $\left.10^{-7}\right)$. The reason for the large difference in the average direction change displayed by RW7000 is also visually apparent. While wild-type worm locomotion occurs in an approximately sinusoidal trajectory, the low fitness RW7000 worms tend to lay still in a much straighter orientation than other strains with shallower wave magnitude during their sinusoidal locomotion. Because their ability to move forward and turn seems to be restricted, any changes in movement tend to be in back-and-forth motion close to $\pi$ radians, while other strains tend to move forward and make slow turns. Direction change showed the following trend: N2/CB4851/RW6999< RW7000.

\section{Local sequence context of Tc1 insertion sites}

Tcl had been well-documented to insert into $5^{\prime}-\mathrm{TA}-3^{\prime}$ target sites with a lightly conserved A/T rich motif (Rosenzweig et al. 1983b; Eide and Anderson 1988). Korswagen et al. (1996) analyzed $83 \mathrm{Tc} 1$ insertion sites to conclude a symmetric consensus sequence for Tc 1 insertion, namely CAYATATRTG. We further analyzed $1,659 \mathrm{Tc} l$ insertion sites in the three Bergerac strains to derive a consensus insertion motif (Figure 5), confirming the conclusions of preceding studies (Eide and Anderson 1988; Korswagen et al. 1996). All the Tcl insertions were located at a 5'-TA-3' target 
594 site within the consensus sequence. The consensus sequence was identified at 46,630

595 locations in the N2 reference genome (PRJNA13758.WS279).

\section{Nonrandom genomic distribution of Tcl elements in the Bergerac strains}

To determine whether Tcl proliferation in the Bergerac strains was random or influenced by genomic context, we classified the $C$. elegans genome based on four broad categories: (i) chromosomes, (ii) recombination domains, (iii) chromatin environment, and (iv) exonic, intronic or intergenic regions.

There is significant variation in chromosomal distribution of $\mathrm{Tc} l$ elements between the three Bergerac strains $\left(G=96.27, p=3.33 \times 10^{-16}\right)$ (Figures 6A and 6B). This is primarily due to the relatively low number of Tcl elements on Chr. V in CB4851. When Chr. V was excluded from the analysis, the chromosomal distribution of Tc 1 was not significantly different between RW6999 and RW7000 $(G=1.07, p=0.96)$, nor between the three Bergerac strains $(G=4.32, p=0.83)$. Additionally, Tcl insertions were nonrandomly distributed across the six chromosomes (five autosomes and X) in the three Bergerac strains once corrected for chromosome length. Tcl elements were overrepresented on Chr. V and X in the closely-related strains RW6999 $\left(\chi^{2}=49.06, p=\right.$ $\left.2.1610^{-9}\right)$ and $\operatorname{RW} 7000\left(\chi^{2}=50.88, p=9.1510^{-10}\right)$. This Tc 1 overrepresentation was particularly pronounced on chromosome $\mathrm{V}$ which had $48 \%$ and $40 \%$ more Tc 1 elements than expected based on chromosome length alone in strains RW6999 and RW7000, respectively. When the number of $8 \mathrm{bp}$ consensus Tc 1 insertion sites $\left(5^{\prime}-\mathrm{AYATATRT}-3^{\prime}\right)$ per chromosome is taken into account, the number of Tc 1 are $56 \%$ and 49\% greater on chromosome V than expected in RW6999 and RW7000, respectively. Tc1 number was better correlated with chromosome size $\left(R^{2}=0.96\right.$ for RW6999) than the number of consensus Tc 1 insertion sites per chromosome $\left(R^{2}=0.70\right.$ for RW6999).

The genome was additionally split into arms, cores, and tips based on recombination domains designated by Rockman and Kruglyak (2009) (Figure 7). Genepoor high-recombination arms, gene-rich low-recombination cores, and gene-poor low- 
recombination tips comprise $45.7 \%, 47 \%$ and $7.3 \%$ of the C. elegans genome, respectively. The distribution of $\mathrm{Tc} l$ insertions in arms and cores was not significantly different from random expectation within N2 $\left(\chi^{2}=2.66, p=0.21\right)$, CB4851 $\left(\chi^{2}=0.47, p\right.$ $=0.49), \operatorname{RW6999}\left(\chi^{2}=4.39, p=0.11\right)$ and $\operatorname{RW7000}\left(\chi^{2}=4.81, p=0.11\right)$ corrected for multiple-comparisons (Holm-Bonferroni method; Holm 1979). Furthermore, there was no significant difference between cores and arms in Tcl insertions that are shared between Bergerac strains and insertions that are unique to a particular strain (Supplemental Figure S2; $G=3.24, p=0.072$ ). Additional analysis of chromosomal tips was excluded because they represent such a small proportion of the genome.

To ascertain transposition into different chromatin environments, five previously identified broad patterns of histone modification (Liu et al. 2011) were used to gate Tcl insertions in the three Bergerac strains relative to the Bristol N2 strain. These five patterns of histone modification are as follows: (i) lowly expressed genes (H3K27me3), (ii) repetitive regions (H3K9me1/2/3), (iii) dosage compensation (H3K27me1, H4K20me1), (iv) promoters of highly expressed genes (H3K4me1/2/3, H3K27ac, H4K8ac, H4K16ac), and (v) highly expressed genes (H3K36me3, H3K79me1/2/3). As there was potential overlap between histone modification context, each category was tested individually for each strain. Interestingly, all Tc1 locations identified in N2, both in the reference genome and in our laboratory isolate, inhabited regions associated with $\mathrm{H} 3 \mathrm{~K} 27 \mathrm{me} 3$ or $\mathrm{H} 3 \mathrm{~K} 9 \mathrm{me} 1 / 2 / 3$, and canonically identified as repressed in the C. elegans genome (Figures 8A and 8B), while being absent from regions associated with high expression (Figures 8C and 8D).

No significant preferences were found in Tcl insertions in domains associated with dosage compensation and enriched on the X chromosome (H3K27me1, H4K20me1) in any of the Bergerac strains (CB4851: $\chi^{2}=0.003, p=1$; RW6999: $\chi^{2}=2.12, p=0.40$; RW7000: $\chi^{2}=0.44, p=1$, Holm-Bonferroni corrected) (not shown in Figure 8). There were no significant differences between the three Bergerac strains with regards to Tc 1 insertions in any of the five chromatin modification regions analyzed here (four shown in Figure 8). In heterochromatic regions associated with H3K27me 3 and low gene 
expression (Figure 8A), Tc 1 insertions were more abundant than expected by chance in $\operatorname{CB} 4851\left(\chi^{2}=7.44, p=0.013\right)$ and RW6999 $\left(\chi^{2}=8.82, p=0.0089\right)$, but not RW7000 $\left(\chi^{2}\right.$ $=2.82, p=0.09$ ) after correcting for multiple comparisons (Holm 1979). Chromatin regions associated with $\mathrm{H} 3 \mathrm{~K} 9 \mathrm{me} 1 / 2 / 3$, repetitive DNA and low gene expression (Figure 8B), were also slightly enriched for Tcl insertions in RW6999 $\left(\chi^{2}=5.06, p=0.049\right)$ and RW7000 $\left(\chi^{2}=7.06, p=0.024\right)$, but not in CB4851 $\left(\chi^{2}=7.06, p=0.44\right)$. Domains associated with H3K36me3, H3K79me1/2/3 and high gene expression (Figure 8D) had fewer Tcl insertions than expected by chance in all three Bergerac strains (CB4851: $\chi^{2}=$ 48.33, $p=3.60 \times 10^{-12}$; RW6999: $\chi^{2}=75.28, p=8.17 \times 10^{-18} ; \mathrm{RW7000}: \chi^{2}=82.71, p=$ $2.85 \times 10^{-19}$, Holm-Bonferroni corrected). Similarly, domains associated with promoters of highly expressed genes (H3K4me1/2/3, H3K27ac, H4K8ac, H4K16ac, Figure 8C) had fewer Tcl insertions than expected by chance (CB4851: $\chi^{2}=10.91, p=0.00287$; RW6999: $\chi^{2}=5.96, p=0.0184$; RW7000: $\chi^{2}=6.79, p=0.0184$, Holm-Bonferroni corrected). Some of the Tcl insertions associated with highly expressed genes in the Bergerac strains were found in germline enriched genes (Han et al. 2019). CB4851, RW6999, and RW7000 harbored 10, 17, and 21 Tc1 insertions within or near highly expressed germline enriched genes, respectively. In sum, repressed chromatin had excess insertions and transcriptionally active chromatin had fewer Tcl insertions than expected by chance. However, Tcl insertions are significantly more likely in transcriptionally active chromatin in the Bergerac strains than in N2 (Fisher's exact test: N2 vs. CB4851, $\left.p_{a d j}=0.0243 ; \mathrm{N} 2 v s . \mathrm{RW} 6999, p_{a d j}=0.0272 ; \mathrm{N} 2 v s . \mathrm{RW} 7000, p_{a d j}=0.0243\right)$.

There were significant differences between the four strains in the distribution of Tcl elements in exons, introns, and intergenic regions $\left(G=54.26, p=6.5 \times 10^{-10}\right)$. None of the Tcl insertions in $\mathrm{N} 2$ were present in exons; rather they appear to be overrepresented in introns (Fischer's exact: $p=1.3 \times 10^{-5}$ ). The distribution of Tcl is not significantly different across exons, introns, and intergenic regions between the three Bergerac strains $(G=4.28, p=0.28)$. However, Tcl appears to be underrepresented in exons and overrepresented in introns and intergenic regions in $\mathrm{RW6999}\left(\chi^{2}=10.20, p=\right.$ 0.012 , adjusted for multiple comparison) and RW7000 $\left(\chi^{2}=22.32, p=5.6 \times 10^{-5}, p\right.$ adjusted by Holm-Bonferroni correction). The underrepresentation of Tcl in exons could 
687 be due to selection. However, potential Tcl insertion sites are also more abundant in

688 introns and intergenic regions due in part to their higher A/T content compared to exons.

689 If we recalculate the expected number of Tc 1 insertions in exons, introns and intergenic

690 regions based on the frequency of the consensus integration sequence in these categories,

691 Tcl insertions are overrepresented in exons and underrepresented in intergenic regions

$692 \quad\left(\mathrm{CB} 4851: \chi^{2}=38.61, p=8.27 \times 10^{-9} ; \mathrm{RW} 6999: \chi^{2}=59.53, p=3.55 \times 10^{-13} ; \mathrm{RW7000}: \chi^{2}\right.$

$693=35.14, p=2.35 \times 10^{-8}, p$ adjusted by Holm-Bonferroni correction). For example, in

694 Bergerac strain RW6999, the expected number of Tcl insertions in exons is 121 based on

695 the frequency of consensus insertions sites whereas the observed number is 190 .

696

\section{Mutations in the Bergerac strains}

Tcl insertions were found to disrupt the exons of many protein-coding genes. Since the insertion of TEs into exons can lead to a disruption or total knock-out of gene function (Orgel and Crick 1980; Muñoz-López and García-Pérez 2010), the RNAi phenotypes for these genes provides a prediction for the effect of these insertions. These RNAi phenotypes (Supplemental Table S10) include many phenotypes observed in the phenotypic assays, including reduced brood size, slow growth, embryonic lethality, shortened life span, and locomotion defects. In addition, the Bergerac strains share 6,191 homozygous SNPs/indels after filtering (see Materials and Methods). These mutations were predicted to result in an amino acid change in 384 genes (Supplemental Table S11) and 122 of these mutations were predicted to have a deleterious effect on protein function by SIFT (Vaser et al. 2016). No candidate mutations were identified in genes known to be relevant for the small RNA pathways linked to regulation of TE activity

(Supplemental Table S11; Billi et al. 2014). However, the protein abt-1 was annotated by WormBase as being involved in germline RNAi. Despite a lack of damage to sRNArelated genes, several genes related to meiosis and recombination (him- $8, \mathrm{msh}-5$, rec-8, $z y g-12$, and $d s b-1$ ) were predicted to have deleterious amino acid changes by SIFT (Vaser et al. 2016). The MiModd package also produced calls for deletions in the Bergerac strains. However, no exonic deletions in genes potentially involved in TE suppression were identified. 
In order to generate divergence time estimates among the Bergerac strains and the N2 (Bristol) strain, we employed WGS data to determine mtDNA genetic divergence (SNPs) between N2 and each of the three focal Bergerac strains. N2-RW7000, N2-RW6999, and N2-CB4851 differed by four SNPs each. We assumed (i) a spontaneous base substitution rate of $4.32 \times 10^{-8} /$ site/generation for the C. elegans mtDNA genome (Konrad et al. 2017), and (ii) three estimates of generation time (14-, 30- and 60-day generation) in wild $C$. elegans which is more apt based on the ecology of the species (Cutter 2008). Assuming a generation time of 14, 30, and 60 days, wild $C$. elegans are expected to have approximately 26, 12, and six generations per year, respectively. Under this range of generation time, all three Bergerac strains are estimated to have diverged from N2 between 129-552 years (14-day generation: 129 years; 30-day generation: 276 years; 60-day generation: 552 years). Finally, to place the Bergerac strains in a phylogenetic context, we ran a maximum likelihood analysis (PhyML) on 11 strains, which included the three Bergerac strains and N2 using both mtDNA and nuclear SNPs. The phylogenetic tree matched the expected relationships, with the French natural isolates JU394 and JU2565 displaying the closest relationship to the Bergerac strains, and the Bergerac strains forming a monophyletic group on the tree (Supplemental Figure S2) (Cook et al. 2017).

\section{DISCUSSION}

The Bergerac and the N2 (Bristol) strains were the first two laboratory isolates of C. elegans and hence, of historical importance in the development of the species as a model organism (Riddle et al. 1997). Laboratory culturing of the Bergerac strain preceded that of N2 by several decades (Nigon 1949; Nigon and Dogherty 1949). However, the Bergerac strain displayed temperature-sensitivity (Fatt and Dogherty 1963), low fertility (Abdulkader and Brun 1980; Lee et al. 2016), and other phenotypic 
748 abnormalities including uncoordinated movement (Emmons et al. 1983; Hodgkin and

749 Doniach 1997) which ultimately led to its supplantation in the laboratory by the more 750 phenotypically robust Bristol N2 strain (Brenner 1974; Sulston and Brenner 1974). A 751 series of well-executed studies in succession over a decade from the late 1970s to late 752 1980s served to elucidate the genetic bases of these phenotypic peculiarities that have since become characteristic of the original Bergerac isolate and its descendants (see Introduction), namely the proliferation of a unique DNA transposon called Tcl (Emmons et al. 1979, 1983; Liao et al. 1983; Eide and Anderson 1985; Egilmez et al. 1995). Sequencing of a Tc 1 element showed it to be 1,610 bp in length (coding potential of 343 aa) with short inverted terminal repeats of 54 bp (Rosenzweig et al. 1983a). Interestingly, all copies of $\mathrm{Tc} l$ in $C$. elegans appeared to be uniform in length and largely conserved in their DNA sequence, suggesting that Tcl coded for products mediating its own transcription (Liao et al. 1983) or that the regulatory mechanism for transposition required a full-length element (Mori et al. 1988). The transposition of Tcl was shown to be strain-specific (Eide and Anderson 1985) with the Bergerac strain BO (RW7000) capable of generating spontaneous mutations in the $u n c-22$ locus at a per generation rate of approximately $10^{-4}$, and three orders of magnitude higher than the baseline spontaneous mutation rate at the same locus in the N2 genetic background (Moerman and Waterston 1984). Furthermore, whilst both the Bergerac (RW7000 subclone) and N2

768 Emmons et al. 1986), the Bergerac strain was unique in having active germline transposition (Collins et al. 1987). It should be mentioned that the history and nomenclature of the Bergerac strains can be confusing given that clones were shared with

771 multiple laboratories over a period of decades and sublineages were often given new names (reviewed in Nigon and Félix 2017). While RW6999 is regarded as a subclone of Bergerac RW7000/BO/LY, the Bergerac strain CB4851 has high Tcl content (Hodgkin

774 and Doniach 1997) but has been reported to lack the active germline transposition of Tc1 775 (Nigon and Félix 2017) as observed in the RW7000 and RW6999 strains (Moerman and 776 Waterston 1984; Eide and Anderson 1985). Despite a decade of sustained focus in the 777 pre-genomics era, a modern genomic analysis of the genomic distribution of TEs, a 
778 precise quantification of their relative fitness, and further investigations into a possible

779 cause for TE deregulation in the Bergerac strains is hitherto lacking.

780

781

782

783

784

The transposable element Tcl was shown to be unique to C. elegans and while present in low copy-number in the majority of $C$. elegans strains examined, the Bergerac strain and its descendants were classified as high copy-number strains. Emmons et al. (1983) were the first to use Southern blotting to estimate genomic Tcl copy-number in the N2 and Bergerac strain RW7000 (also referred to as the BO subclone) as $20 \pm 5$ and $200 \pm 50$, respectively. Liao et al. (1983) estimated Tc1 copy-number in the N2 and Bergerac strain RW7000 (also referred to as the LY subclone) to be 25-30 and several hundred copies, respectively. Eide and Anderson (1985) mention Bergerac RW7000/BO/LY lineage as possessing $250 \mathrm{Tc} 1$ copies as per Emmons et al. (1983) and Liao et al. (1983). Mori et al. (1988) subsequently found the RW7000/BO/LY lineage to harbor $>300 \mathrm{Tc} 1$ copies. Hence, Southern blotting techniques established a range of 2530 and 200 to $>300$ Tc 1 copies for the N2 and RW7000 strain, respectively. Recognizing the limitation of Southern blots in the accurate estimation of Tcl in high-copy strains, Egilmez et al. (1995) opted to use DNA dot-blots. N2 and Bergerac RW7000/BO/LY were estimated to possess 31 and $515 \pm 48 \mathrm{Tc} 1$ copies, respectively (Egilmez et al. 1995). More recently, Larichhia et al. (2017) were the first to estimate transposon abundance in several hundred $C$. elegans isolates from Illumina pair-end sequencing data. One Bergerac sublineage, CB4851, was included in their study and estimated to possess 406 Tc 1 copies (Laricchia et al. 2017). However, because there are no existing previous hybridization-based estimates of Tcl count for CB4851, it is not possible to compare transposon number estimates generated from hybridization versus WGS data. This study offers new estimates of Tcl counts for three Bergerac strains based on both (i) bioinformatic analyses of WGS data, and (ii) independent confirmation by ddPCR. We determined far higher copy-number estimates of Tcl in the Bergerac strains than preceding studies with median values of 451, 581 and 748 copies for CB4851, RW6999, and RW7000, respectively. In the best-studied RW7000 Bergerac strain, our estimate of Tc 1 copy-number is more than $2 \times$ greater than past studies relying on Southern hybridizations (Emmons et al. 1983; Liao et al. 1983; Mori et al. 1988) and 
approximately $1.5 \times$ greater than an estimate based on DNA dot-blots (Egilmez et al. 1995). The discrepancy in our estimates of Tcl copy-number and those of past studies relying on Southern hybridizations and DNA dot-blots is likely due to drawbacks associated with the latter techniques, namely membrane saturation limiting resolution of copy-number when counts exceed $\sim 100$. The five bioinformatic methods employed to estimate TE copy-number were consistent with each other, both in the number of calls per strain and in the differences in Tcl copy-number between the strains. Furthermore, $90 \%$ and $70 \%$ of the calls were shared by more than one method and by all methods, respectively. Nevertheless, these median numbers of Tcl are likely to be underestimates as both sequence read coverage and ddPCR yielded even higher estimates of Tcl copy number in the three Bergerac strains.

Both our ddPCR and bioinformatic approaches demonstrate that Tc 1 copynumber can vary considerably among the three Bergerac sublineages/strains examined in this study (median values of 451, 581 and 748 copies for CB4851, RW6999, and RW7000, respectively). This among-strain variation in Tcl copy-number could be owing to either one or a combination of at least two factors. First, it is possible that some Bergerac sublineages have evolved a mechanism of TE regulation that limits further proliferation of Tc1. For example, CB4851 is thought to lack the active germline transposition characteristic of the RW7000 and RW6999 strains (Nigon and Félix 2017). Second, the complete history of laboratory propagation for these strains over multiple decades is obscure, with some strains possibly having been subjected to lengthier periods of cryopreservation or laboratory evolution than others.

Aside from Tcl, the only consistent difference in TE copy-number between the Bergerac strains and N2 was for Tc2. The average number of Tc2 elements was 20 and three in the Bergerac strains and N2, respectively, and normalized read depth suggests 25-30 copies of Tc2 in the Bergerac strains and four in N2. Tc2, originally discovered in a Bergerac strain, was upon its discovery found in higher copy-number Tcl strains than in low-copy number Tcl strains (Levitt and Emmons 1989). This coincidence suggested that the proliferation of Tc 1 and Tc 2 was due to shared copy-number regulation 
840 mechanisms (Levitt and Emmons 1989). Since then, many genes have been discovered in

841 C. elegans that influence the transposition of multiple TEs (Vastenhouw et al. 2003; Lee

842 et al. 2012; Weick and Miska 2014; McMurchy et al. 2017; Wallis et al. 2019). In

843 contrast to the striking differences among the Bergerac strains with respect to the number

844 of Tc1 insertions, Tc2 copy-number and locations are remarkably well-conserved. It

845 appears that the explosive proliferation and divergence of $\mathrm{Tc} l$ insertions in the Bergerac

846 strains is caused by factors specific to Tcl and not shared with other TEs in C. elegans. (Wood et al. 1980; Emmons et al. 1983), this study is the first to offer precise quantification of their low composite fitness via phenotypic assays of four fitness-related traits (productivity, survivorship to adulthood, developmental rate, and longevity).

Notably, all three Bergerac strains differ significantly from N2 with respect to each of the four fitness traits; in each case, their mean fitness trait values are far lower than N2. Productivity and survivorship to adulthood are two traits considered most germane to organismal fitness. The three Bergerac strains exhibited an extensive reduction $(65-86 \%)$ in productivity relative to wild type N2. To place this in perspective, spontaneous mutation accumulation (MA) lines of C. elegans subjected to extreme genetic drift and inbreeding for $>400$ consecutive generations displayed an average $44-55 \%$ reduction in productivity (Katju et al. 2015, 2018). Mean survivorship to adulthood in the three Bergerac strains was reduced by $7-15 \%$ relative to wild type N2. In contrast to productivity, this reduction in survivorship of the Bergerac strains is on par with the 12$19 \%$ decline observed in the same long-term spontaneous MA lines of C. elegans evolved over 400 generations (Katju et al. 2015, 2018). The Bergerac strains also exhibited reduced longevity (29-36\% lower relative to N2) as well as delayed developmental rate (9-20\% longer than N2). These experiments do not bear on the question whether TE proliferation alone directly contributes to all observable fitness decline in the Bergerac strains. Indeed, an independent mutation in the zyg-12 gene in the Bergerac strain renders it almost completely sterile at $25^{\circ} \mathrm{C}$ (Malone et al. 2003). However, TE mobilization events have been shown to have a direct negative impact on host fitness; notable examples include the reduction of host fertility and viability following $P$-element 
871 invasion in Drosophila (Kidwell and Novy 1979), decreased fitness and egg hatchability

872 in laboratory lines of D. melanogaster with elevated TE transposition rates (Pasyukova et

873 al. 2004), and fitness loss in Drosophila mutation accumulation lines following copia

874 insertions (Houle and Nuzhdin 2004). It is therefore reasonable to assume that the low

875 fitness of the Bergerac strain and its derivatives is owing largely to the unchecked

876 proliferation of TEs.

877

In addition to confirming that all Bergerac strains had significantly lower fitness than N2, our study also quantified significant inter-strain differences among the three Bergerac strains studied here with respect to fitness. RW7000 (or Bergerac-BO), used in many transposon-tagging studies in the 1990s (Korswagen et al. 1996), exhibited the lowest means for three traits (productivity, and survivorship, and longevity). RW7000 also possesses the highest Tcl copy-number among the three strains studied here. Interestingly, the strain RW6999, listed as an RW subclone of RW7000 by the Caenorhabditis Genetics Center (CGC), displayed the highest fitness among the Bergerac strains, despite having the second-highest number of Tcl insertions. The hypothesis that TE proliferation is the sole cause of fitness differences between the three Bergerac strains is ruled out by these results. It appears that fitness is not simply correlated with copynumber of active Tcl elements but other determinants as well such as site(s) of genomic integration, and/or certain key loci with high mutator activity.

Bergerac strains are also known to display abnormal phenotypes with respect to locomotion, and are often referred to as "uncoordinated" (Brenner 1974; Hodgkin and Doniach 1997; Emmons et al. 2003; Nigon and Félix 2017). The unc-22 gene on Chr. IV plays an important role in muscle structure and function (Moerman et al. 1988), and is a frequent target for Tcl element insertions in the Bergerac strains (Moerman et al. 1986). As was the case with fitness-related traits, all three Bergerac strains had significantly reduced mean body length (16-20\% reduction) and body area (17-31\%) relative to N2. In addition, some Bergerac strains exhibit significant deviations from N2 with respect to locomotion. RW6999 and RW7000 have significant reductions in speed (32\% and 70\%, respectively) relative to $\mathrm{N} 2$. In addition, RW7000 worms displayed a compromised 
902 ability to move forward and turn, resulting in a significant increase in direction change

$903(113 \%)$ relative to N2. Hence, it appears that RW7000 worms have the most severe

904 impairment in locomotion relative to N2. It is likely that Tc1 insertions have contributed

905 to the observed changes in these quantitative traits in conjunction with other independent

906 mutations in the unique evolutionary trajectory of the Bergerac ancestor and its derived

907 lineages. One mutation present in all three Bergerac strains was a nonsynonymous base

908 substitution in an olfactory G-protein-coupled receptor (GPCR) str-208 yielding a

909 Cys226Ser/Tyr. This mutation may result in improper folding of the protein, with

910 possible inhibitory effects on proper signaling and sensation of external stimuli. The

911 inability to properly relay/convey external stimuli could explain the erratic movement

912 exhibited by the Bergerac strains in the motility assays. The Bergerac strains were

913 additionally noted to leave the area of the bacterial feeding lawn or position themselves

914 around the bacterial seed instead of entering to feed. This behavior could be rooted in a

915 dysfunctional olfactory system and possibly contribute to diminished fitness. necessary for function as well as repetitive DNA sequences that may not contribute to organismal function. This latter component, which may constitute a large fraction of eukaryotic genomes, includes TEs that can be detrimental to the host genome when they transpose into coding regions thereby causing gene disruption. The genome has therefore been likened to an ecological community with TEs being functionally regarded as parasitic invaders with the host genome under selective pressure to actively engage in tackling this TE spread (Brookfield 2005; Bourque et al. 2018). Hence, the distribution and abundance of TEs across the genome is frequently nonrandom due to a combination of natural selection, as well as TE integration site preferences, and deletions (Bourque et al. 2018). TEs are a source of both germline and somatic mutations, contribute to ectopic recombination and can interfere with normal gene expression, all of which are, on average, deleterious to host fitness. Consequently, TEs are observed less frequently within coding and regulatory DNA sequences than expected by chance. Furthermore, the

932 effects of natural selection against both ectopic exchange and insertional mutations. For 
example, the numbers of DNA-based transposable elements are negatively correlated with recombination rate in the Drosophila melanogaster genome (Rizzon et al. 2002). Assuming that opportunities for ectopic recombination between TEs increase with the local recombination frequency, the presence of TEs is more likely to be detrimental in regions with high recombination rates compared to low recombination rates (Langley et al. 1988). Moreover, natural selection is less efficient in regions with low recombination relative to high recombination which predicts that deleterious TE insertions are more likely to accumulate in regions of low recombination (Hill and Robertson, 1966; Dolgin and Charlesworth 2008). In addition, insertional preferences may vary with non-random distribution of features such as suitable integration motifs, recombination rate, and chromatin organization (Sultana et al. 2017). Tcl transpositions in C. elegans have been shown to occur more frequently both (i) within than between chromosomes, and (ii) to proximate than distant locations on the same chromosome (Fischer et al. 2003). Consequently, this bias towards proximate locations violates the usual assumption of independence in statistical tests. If, for now, we ignore these limitations to interpreting the genome-wide distribution of Tcl elements, we find that Tcl is overrepresented on Chr. V in strains RW6999 and RW7000, and on the X chromosome in all three Bergerac strains after correcting for chromosome length. In C. elegans, the chromosome arms are relatively gene-poor regions but have higher recombination frequency than chromosome cores, which are also gene-rich (Rockman and Kruglyak, 2009). In contrast to the relationship between recombination rate and TEs in Drosophila, TE abundance in $C$. elegans is greater in the high-recombination arms than in the low-recombination cores (Duret et al. 2000; Laricchia et al. 2017). We did not find a significant difference in the abundance of $\mathrm{Tc} l$ elements between the chromosome arms and cores across the three Bergerac strains analyzed here. In this regard, our results are similar to an earlier analysis of TEs in C. elegans that also failed to find a significant relationship between recombination rate and Tcl copy-number (Duret et al. 2000).

Tcl is underrepresented in exons which is consistent with selection against strongly deleterious insertions in coding regions of the genome. However, the consensus Tcl integration motif is more commonly found in introns and intergenic regions. The 
964 underrepresentation of Tcl abundance in exons could therefore primarily be a function of

965 the availability of suitable integration motifs. In a similar vein, Tcl insertions in the

966 Bergerac strains were slightly less and more abundant than expected by chance in

967 transcriptionally active and repressed chromatin, respectively. This pattern could arise by

968 insertional preference into repressed chromatin, unequal distribution of integration motifs

969 between repressed and transcriptionally active chromatin, or selection against insertions

970 into transcriptionally active chromatin. Indeed, there is strong purifying selection on TE

971 expression in experimental populations of C. elegans (Bergthorsson et al. 2020).

972 Furthermore, there is a striking difference between the Bristol N2 and the Bergerac

973 strains in that the former sequenced for this study retain Tc 1 insertions exclusively in

974 repressive chromatin. The Bergerac strains contained none of the alleles that have

975 previously been found to derepress Tcl activity (Vastenhouw et al. 2003; Lee et al. 2012;

976 Weick and Miska 2014; McMurchy et al. 2017; Wallis et al. 2019) and mapping putative

977 mutator loci for Tcl in these strains suggested that the mutator activity was associated

978 with multiple sites in the genome which were themselves mobile (Moerman and

979 Waterston 1984; Mori et al. 1988). The presence of numerous Tcl elements insertions in

980 transcriptionally active chromatin in the Bergerac strains could explain their high rate of

981 germline transposition. Even if Tcl preferentially integrates into repressed chromatin,

982 thus evading purifying selection to some degree, serendipitous insertions of Tcl into an

983 active chromatin environment could create a proliferative loop of Tc 1 expansion which

984 selection is simply unable to completely restrain.

The evolutionary history of the Bergerac strain and its derivatives remains

988 Bergerac, France in 1944 (Nigon and Félix 2017). How evolutionary diverged are the

989 Bergerac and the N2 strains? Both earlier and recent studies of C. elegans intraspecific

990 genetic diversity have similarly observed that single-nucleotide variant (SNV) diversity is

991 often shared among many natural isolates and that the species is characterized by lower

992 levels of genetic diversity relative to other obligately outcrossing as well as facultatively

993 selfing species in the genus Caenorhabditis (Denver et al. 2003; Sivasundar and Hey

994 2003; Cutter 2006; Dey et al. 2013; Thomas et al. 2015). Based on mtDNA genetic 
995 divergence among the three Bergerac strains and N2 and assuming three different

996 estimates of generation time in the wild, we estimated a very evolutionary recent N2-

997 Bergerac divergence range of 129-552 years. As expected, the three focal Bergerac

998 strains in this study form a monophyletic clade and are more closely related to two

999 French natural isolates, providing evidence of some biogeographic structure.

1000 Another pertinent outstanding question is whether the high activity of Tc 1 transposition

1001 in the Bergerac germline originated in the wild or occurred following laboratory

1002 domestication. Interestingly, the subclones/derivatives of Bergerac display variation with

1003 respect to active germline transposition of Tcl (Emmons et al. 1983; Liao et al. 1983;

1004 Moerman and Waterston 1984). For example, the BO/RW7000 sublineage has active

1005 germline transposition (Moerman and Waterston 1984), extremely elevated Tcl copy-

1006 number and drastically reduced fitness (latter two features confirmed in this study),

1007 whereas CB4851 has a high transposon content (though not as elevated as BO/RW7000

1008 and RW6999) but is thought to lack active germline transposition of Tcl (Nigon and

1009 Félix 2017). What evolutionary scenario would explain the active germline transposition

1010 of Tcl in some Bergerac sublineages and not others? Under one scenario, the wild

1011 ancestor of all Bergerac strains possessed active germline transposition of Tcl which has

1012 been suppressed (selection) or lost (genetic drift) in some Bergerac derivatives following

1013 laboratory domestication. A second scenario to be considered is the activation of Tc1

1014 germline transposition after introduction to the laboratory, sometime in the period

1015 between 1944 and 1969 (see schematic of tentative history of the Bergerac strain in

1016 Nigon and Félix 2017) followed by subsequent suppression in different Bergerac

1017 lineages. It is conceivable that laboratory selection for novel mutations resulted in the

1018 preferential retention of sublineages of Bergerac that generated more mutations due to

1019 germline Tcl activity. The question of how Tclproliferation in the Bergerac was initiated

1020 is still open.

\section{ACKNOWLEDGMENTS}

1025 This research was supported by start-up funds from the Department of Veterinary 
bioRxiv preprint doi: https://doi.org/10.1101/2022.02.02.478770; this version posted February 4, 2022. The copyright holder for this preprint (which was not certified by peer review) is the author/funder, who has granted bioRxiv a license to display the preprint in perpetuity. It is made available under aCC-BY 4.0 International license.

1026 Integrative Biosciences, College of Veterinary Medicine and Biomedical Sciences at

1027 Texas A\&M University to V.K. and U.B. 


\section{LITERATURE CITED}

Abdulkader, N., and J. Brun, 1980 A temperature-sensitive mutant of Caenorhabditis elegans var. Bergerac affecting morphological and embryonic development. Genetics 51: 81-92. https://doi.org/10.1007/bf00133506

Adrion, J. R., M. J. Song, D. R. Schrider, M. W. Hahn, and S. Schaack, 2017 Genome-wide estimates of transposable element insertion and deletion rates in Drosophila melanogaster. Genome Biol. Evol. 9: 1329-1340. https://doi.org/10.1093/gbe/evx050

Almeida, M. V., M. A. Andrade-Navarro, and R. F. Ketting, 2019 Function and evolution of nematode RNAi pathways. Noncoding RNA 5: 8. https://doi.org/10.3390/ncrna5010008

Ambros, V., R. C. Lee, A. Lavanway, P. T. Williams, and D. Jewell, 2003 MicroRNAs and other tiny endogenous RNAs in C. elegans. Curr. Biol. 13: 807-818. https://doi.org/10.1016/s0960-9822(03)00287-2

Angstman, N. B., H. Frank, and C. Schmitz, 2016 Advanced behavioral analyses show that the presence of food causes subtle changes in C. elegans movement. Front. Behav. Neurosci. 10: 1-10. https://doi.org/10.3389/fnbeh.2016.00060

Ashe, A., A. Sapetschnig, E.-M. Weick, J. Mitchell, M. P. Bagijn et al., 2012 piRNAs can trigger a multigenerational epigenetic memory in the germline of C. elegans. Cell 150: 88-99. https://doi.org/10.1016/j.cell.2012.06.018

Bagijn, M. P., L. D. Goldstein, A. Sapetschnig, E. M. Weick, S. Bouasker et al., 2012 Function, targets, and evolution of Caenorhabditis elegans piRNAs. Science 337: 574-578. https://doi.org/10.1126/science.1220952

Batista, P. J., J. G. Ruby, J. M. Claycomb, R. Chiang, N. Fahlgren et al., 2008 PRG-1 and 21URNAs interact to form the piRNA complex required for fertility in C. elegans. Mol. Cell 31: 67-78. https://doi.org/10.1016/j.molcel.2008.06.002

Beltran, T., V. Shahrezaei, V. Katju, and P. Sarkies, 2020 Epimutations driven by small RNAs arise frequently but most have limited duration in Caenorhabditis elegans. Nat. Ecol. Evol. 4: 1539-1548. https://doi.org/10.1038/s41559-020-01293-z

Bergthorsson, U., C. J. Sheeba, A. Konrad, T. Belicard, T. Beltran et al., 2020 Long-term experimental evolution reveals purifying selection on piRNA-mediated control of 
transposable element expression. BMC Biol. 18: 162. https://doi.org/10.1186/s12915020-00897-y

Billi, A. C., S. E. Fischer, and J. K. Kim, 2014 Endogenous RNAi pathways in C. elegans.

WormBook May 7: 1-49. https://doi.org/10.1895/wormbook.1.170.1

Blumenstiel, J. P., 2011 Evolutionary dynamics of transposable elements in a small RNA world.

Trends Genet. 27: 23-31. https://doi.org/10.1016/j.tig.2010.10.003

Bourque, G., K. H. Burns, M. Gehring, V. Gorbunova, A. Seluanov et al., 2018 Ten things you should know about transposable elements. Genome Biol. 19: 199. https://doi.org/10.1186/s13059-018-1577-z

Brenner. S., 1974 The genetics of Caenorhabditis elegans. Genetics 77:71-94. https://doi.org/10.1093/genetics/77.1.71

Brookfield, J. F. Y., 2005 The ecology of the genome - mobile DNA elements and their hosts. Nat. Rev. Genet. 6: 128-136. https://doi.org/10.1038/nrg1524

Byerly, L., R. C. Cassada, and R. L. Russell, 1976 The life cycle of the nematode Caenorhabditis elegans: I. Wild-type growth and reproduction. Dev. Biol. 51: 23-33. https://doi.org/10.1016/0012-1606(76)90119-6

C. elegans Sequencing Consortium, 1998 Genome sequence of the nematode C. elegans: a platform for investigating biology. Science 282: 2012-2018. https://doi.org/10.1126/science.282.5396.2012

Chuong, E. B., N. C. Elde, and C. Feschotte, 2017 Regulatory activities of transposable elements: from conflicts to benefits. Nat. Rev. Genet. 18: 71-86. https://doi.org/10.1038/nrg.2016.139

Cingolani, P., A. Platts, L. L. Wang, M. Coon, T. Nguyen, et al., 2012 A program for annotating and predicting the effects of single nucleotide polymorphisms, SnpEff: SNPs in the genome of Drosophila melanogaster strain w1118; iso-2; iso-3. Fly 6: 80-92. https://doi.org/10.4161/fly.19695

Collins, J., B. Saari, and P. Anderson, 1987 Activation of a transposable element in the germ line but not the soma of Caenorhabditis elegans. Nature 328: 726-728. https://doi.org/10.1038/328726a0

Cook, D. E., S. Zdraljevic, J. P. Roberts, and E. C. Andersen, 2017 CeNDR, the Caenorhabditis 
elegans natural diversity resource. Nucleic Acids Res. 45: 650-657.

https://doi.org/10.1093/nar/gkw893

Cutter, A. D., 2006 Nucleotide polymorphism and linkage disequilibrium in wild populations of the partial selfer Caenorhabditis elegans. Genetics 172:171-184. https://doi.org/10.1534/genetics.105.048207

Cutter, A. D., 2008 Divergence times in Caenorhabditis and Drosophila inferred from direct estimates of the neutral mutation rate. Mol. Biol. Evol. 25: 778-786. https://doi.org/10.1093/molbev/msn024

Das, P. P., M. P. Bagijn, L. D. Goldstein, J. R. Woolford, N. J. Lehrbach et al., 2008 Piwi and piRNAs act upstream of an endogenous siRNA pathway to suppress Tc3 transposon mobility in the Caenorhabditis elegans germline. Mol. Cell 31: 79-90. https://doi.org/10.1016/j.molcel.2008.06.003

Denver, D. R., K. Morris, and W. K. Thomas, 2003 Phylogenetics in Caenorhabditis elegans: an analysis of divergence and outcrossing. Mol. Biol. Evol. 20: 393-400. https://doi.org/10.1093/molbev/msg044

Dey, A., C. K. W. Chan, C. G. Thomas, and A. D. Cutter, 2013 Molecular hyperdiversity defines populations of the nematode Caenorhabditis brenneri. Proc. Natl. Acad. Sci. USA 110: 11056-11060. https://doi.org/10.1073/pnas.1303057110

Dolgin, E. S., and B. Charlesworth, 2008 The effects of recombination rate on the distribution and abundance of transposable elements. Genetics 178: 2169-2177. https://doi.org/10.1534/genetics.107.082743

Dubie, J. J., A. R. Caraway, M. K. M. Stout, V. Katju, and U. Bergthorsson, 2020 The conflict within: origin, proliferation and persistence of a spontaneously arising selfish mitochondrial genome. Phil. Trans. Royal Soc. B 375: 20190174. https://doi.org/10.1098/rstb.2019.0174

Duret, L., G. Marais, and C. Biémont, 2000 Transposons but not retrotransposons are located preferentially in regions of high recombination rate in Caenorhabditis elegans. Genetics 156:1661-1669. https://doi.org/10.1093/genetics/156.4.1661

Egilmez, N. K., R. H. Ebert 2nd, and R. J. Shmookler Reis, 1995 Strain evolution in Caenorhabditis elegans: transposable elements as markers of interstrain evolutionary history. J. Mol. Evol. 40: 372-381. https://doi.org/10.1007/BF00164023 
Eide, D., and P. Anderson, 1985 Transposition of Tcl in the nematode Caenorhabditis elegans.

Proc. Natl. Acad. Sci. USA 82: 1756-1760. https://doi.org/10.1073/pnas.82.6.1756

Eide, D., and P. Anderson, 1988 Insertion and excision of Caenorhabditis elegans transposable element Tc1. Mol. Cell. Biol. 8: 737-746. https://doi.org/10.1128/mcb.8.2.737-746.1988

Emmons, S. W., M. R. Klass, and D. Hirsh, 1979 Analysis of the constancy of DNA sequences during development and evolution of the nematode Caenorhabditis elegans. Proc. Natl. Acad. Sci. USA 76: 1333-1337. https://doi.org/10.1073/pnas.76.3.1333

Emmons, S. W., S. Roberts, and K. S. Ruan, 1986 Evidence in a nematode for regulation of transposon excision by tissue-specific factors. Mol. Gen. Genet. 202: 410-415. https://doi.org/10.1007/BF00333270.

Emmons, S. W., and L. Yesner, 1984 High-frequency excision of transposable element Tcl in the nematode Caenorhabditis elegans is limited to somatic cells. Cell 36: 599-605. https://doi.org/10.1016/0092-8674(84)90339-8

Emmons, S. W., L. Yesner, K. S. Ruan, and D. Katzenberg, 1983 Evidence for a transposon in Caenorhabditis elegans. Cell 32: 55-65. https://doi.org/10.1016/0092-8674(83)90496-8

Fatt, H. V., and E. C. Dougherty, 1963 Genetic control of differential heat tolerance in two strains of the nematode Caenorhabditis elegans. Science 141: 266-267. https://doi.org/10.1126/science.141.3577.266

Files, J. G., S. Carr, and D. Hirsh, 1983 Actin gene family of Caenorhabditis elegans. J. Mol. Biol. 164: 355-375. https://doi.org/10.1016/0022-2836(83)90056-6

Fischer, S. E. J., E. Wienholds, and R. H. A. Plasterk, 2003 Continuous exchange of sequence information between dispersed $\mathrm{Tc} 1$ transposons in the Caenorhabditis elegans genome. Genetics 164: 127-134. https://doi.org/10.1093/genetics/164.1.127

Guindon, S., J. F. Dufayard, V. Lefort, M. Anisimova, W. Hordijk et al., 2010 New algorithms and methods to estimate maximum-likelihood phylogenies: assessing the performance of PhyML 3.0. Syst. Biol. 59: 307-321. https://doi.org/10.1093/sysbio/syq010

Han, M., G. Wei, C. E. McManus, L. W. Hillier, and V. Reinke, 2019 Isolated C. elegans germ nuclei exhibit distinct genomic profiles of histone modification and gene expression. BMC Genomics 20:500. https://doi.org/10.1186/s12864-019-5893-9

Han, S., P. J. Basting, G. B. Dias, A. Luhur, A. C. Zelhof et al., 2021 Transposable element 
profiles reveal cell line identity and loss of heterozygosity in Drosophila cell culture.

Genetics 219: iyab113. https://doi.org/10.1093/genetics/iyab113

Hill, W. G., and A. Robertson, 1966 The effect of linkage on limits to artificial selection.

Genet. Res. 8: 269-294.

Hodgkin, J., and T. Doniach, 1997 Natural variation and copulatory plug formation in Caenorhabditis elegans. Genetics 146: 149-164.

https://doi.org/10.1093/genetics/146.1.149

Holm, S., 1979 A simple sequentially rejective multiple test procedure. Scand. J. Stat. 6: 65-70.

Houle, D., and S. V. Nuzhdin, 2004 Mutation accumulation and the effect of copia insertions in Drosophila melanogaster. Genet. Res. 83: 7-18. https://doi.org/10.1017/s0016672303006505

Katju, V., L. B. Packard, L. Bu, P. D. Keightley, and U. Bergthorsson, 2015 Fitness decline in spontaneous mutation accumulation lines of Caenorhabditis elegans with varying effective population sizes. Evolution 69: 104-116. https://doi.org/10.1111/evo.12554

Katju, V., L. B. Packard, and P. D. Keightley, 2018 Fitness decline under osmotic stress in Caenorhabditis elegans populations subjected to spontaneous mutation accumulation at varying population sizes. Evolution 72: 1000-1008. https://doi.org/10.1111/evo.13463

Keane, T. M., K. Wong, and D. J. Adams, 2013 RetroSeq: transposable element discovery from next-generation sequencing data. Bioinformatics 29: 389-390. https://doi.org/10.1093/bioinformatics/bts697

Kidwell. M. G., and J. B. Novy, 1979 Hybrid dysgenesis in Drosophila melanogaster: sterility resulting from gonadal dysgenesis in the P-M system. Genetics 92: 1127-1140. https://doi.org/10.1093/genetics/92.4.1127

Klein, S. J., and R. J. O'Neill, 2018 Transposable elements: genome innovation, chromosome diversity, and centromere conflict. Chromosome Res. 26: 5-23. https://doi.org/10.1007/s10577-017-9569-5

Konrad, A., S. Flibotte, J. Taylor, R. H. Waterston, D. G. Moerman et al., 2018 Mutational and transcriptional landscape of spontaneous gene duplications and deletions in Caenorhabditis elegans. Proc. Natl. Acad. Sci. USA 115: 7386-7391. https://doi.org/10.1073/pnas.1801930115

Konrad, A., O. Thompson, R. H. Waterston, D. G. Moerman, P. D. Keightley et al., 2017 
Mitochondrial mutation rate, spectrum and heteroplasmy in Caenorhabditis elegans spontaneous mutation accumulation lines of differing population size. Mol. Biol. Evol. 34: 1319-1334. https://doi.org/10.1093/molbev/msx051

Korswagen, H. C., R. M. Durbin, M. T. Smits, and R. H. Plasterk, 1996 Transposon Tc1derived, sequence-tagged sites in Caenorhabditis elegans as markers for gene mapping. Proc. Natl. Acad. Sci. USA 93: 14680-14685. https://doi.org/10.1073/pnas.93.25.14680

Langley, C. H., E. Montgomery, R. Hudson, N. Kaplan, and B. Charlesworth, 1988 On the role of unequal exchange in the containment of transposable element copy number. Genet. Res. 52: 223-235. https://doi.org/10.1017/s0016672300027695.

Laricchia, K. M., S. Zdraljevic, D. E. Cook, and E. C. Andersen, 2017 Natural variation in the distribution and abundance of transposable elements across the Caenorhabditis elegans species. Mol. Biol. Evol. 34: 2187-2202. https://doi.org/10.1093/molbev/msx155

Lee, H.-C., W. Gu, M. Shirayama, E. Youngman, D. Conte Jr. et al. 2012 C. elegans piRNAs mediate the genome-wide surveillance of germline transcripts. Cell 150: 78-87. https://doi.org/10.1016/j.cell.2012.06.016

Lee, Y., W. Hwang, J. Jung, S. Park, J. J. T. Cabatbat et al., 2016 Inverse correlation between longevity and developmental rate among wild C. elegans strains. Aging 8: 986-999. https://doi.org/10.18632/aging.100960

Levitt, A., and S. W. Emmons, 1989 The Tc2 transposon in Caenorhabditis elegans. Proc. Natl. Acad. Sci. USA 86: 3232-3236. https://doi.org/10.1073/pnas.86.9.3232

Liao, L. W., B. Rosenzweig, and D. Hirsh, 1983 Analysis of a transposable element in Caenorhabditis elegans. Proc. Natl. Acad. Sci. USA 80: 3585-3589. https://doi.org/10.1073/pnas.80.12.3585

Lipinski, K. J., J. C. Farslow, K. A. Fitzpatrick, M. Lynch, V. Katju et al., 2011 High spontaneous rate of gene duplication in Caenorhabditis elegans. Curr. Biol. 21: 306-310. https://doi.org/10.1016/j.cub.2011.01.026

Liu, T., A. Rechtsteiner, T. A. Egelhofer, A. Vielle, I. Latorre et al., 2011 Broad chromosomal domains of histone modification patterns in C. elegans. Genome Res. 21: 227-236. https://doi.org/10.1101/gr.115519.110

Lynch, M., 1985 Spontaneous mutations for life-history characters in an obligate parthenogen. Evolution 39: 804-818. https://doi.org/10.1111/j.1558-5646.1985.tb00422.x 
Malone, C. J., L. Misner, N. Le Bot, M.-C. Tsai, J. M. Campbell et al., 2003 The C. elegans hook protein, ZYG-12, mediates the essential attachment between the centrosome and nucleus. Cell 115: 825-836. https://doi.org/10.1016/s0092-8674(03)00985-1

McClintock, B., 1950 The origin and behavior of mutable loci in maize. Proc. Natl. Acad. Sci. USA 36: 344-355. https://doi.org/10.1073/pnas.36.6.344

McMurchy, A. N., P. Stempor, T. Gaarenstroom, B. Wysolmerski, Y. Dong et al., 2017 A team of heterochromatin factors collaborates with small RNA pathways to combat repetitive elements and germline stress. Elife. 6: e21666. https://doi.org/10.7554/eLife.21666

Moerman, D. G., G. M. Benian, R. J. Barstead, L. A. Schriefer, and R. H. Waterston, 1988 Identification and intracellular localization of the unc-22 gene product of Caenorhabditis elegans. Genes Dev. 2:93-105. https://doi.org/10.1101/gad.2.1.93

Moerman, D. G., G. M. Benian, and R. H. Waterston, 1986 Molecular cloning of the muscle gene unc-22 in Caenorhabditis elegans by Tcl transposon tagging. Proc. Natl. Acad. Sci. USA 83: 2579-2583. https://doi.org/10.1073/pnas.83.8.2579

Moerman, D. G., and R. H. Waterston, 1984 Spontaneous unstable unc-22 IV mutations in $C$. elegans var. Bergerac. Genetics 108: 859-877. https://doi.org/10.1093/genetics/108.4.859

Mori, I., D. G. Moerman, and R. H. Waterston, 1988 Analysis of a mutator activity necessary for germline transposition and excision of Tc 1 transposable elements in Caenorhabditis elegans. Genetics 120: 397-407. https://doi.org/10.1093/genetics/120.2.397

Muñoz-López, M., and J. L. García-Pérez, 2010 DNA transposons: nature and applications in genomics. Curr. Genomics 11: 115-128. https://doi.org/10.2174/138920210790886871

Nelson, M. G., R. S. Linheiro, and C. M. Bergman, 2017 McClintock: an integrated pipeline for detecting transposable element insertions in whole-genome shotgun sequencing data. G3 (Bethesda) 7: 2763. https://doi.org/10.1534/g3.117.043893

Nicholas, W. L., E. C. Dougherty, and E. L. Hansen, 1959 Axenic cultivation of C. briggsae (Nematoda: Rhabditidae) with chemically undefined supplements; comparative studies with related nematodes. Ann. N.Y. Acad. Sci. 77: 218-236. https://doi.org/10.1111/j.1749-6632.1959.tb36902.x

Nigon, V., 1949 Les modalités de la reproduction et le déterminisme du sexe chez quelques nematodes libres. Ann. Sci. Nat. Zool. Biol. Anim. 11: 1-132. 
Nigon, V., and E. C. Dougherty, 1949 Reproductive patterns and attempts at reciprocal crossing of Rhabditis elegans Maupas, 1900, and Rhabditis briggsae Dougherty and Nigon, 1949 (Nematoda: Rhabditidae). J. Exp. Zool. 112: 485-503. https://doi.org/10.1002/jez.1401120307

Nigon, V. M., and M.-A. Félix, 2017 History of research on C. elegans and other free-living nematodes as model organisms. WormBook 2017: 1-84. https://doi.org/10.1895/wormbook.1.181.1

Orgel, L. E., and F. H. Crick, 1980 Selfish DNA: the ultimate parasite. Nature 284: 604-607. https://doi.org/10.1038/284604a0

Pasyukova, E. G., S. V. Nuzhdin, T. V. Morozova, and T. F. C. Mackay, 2004 Accumulation of transposable elements in the genome of Drosophila melanogaster is associated with a decrease in fitness. J. Heredity 95: 284-290. https://doi.org/10.1093/jhered/esh050

Payer, L. M., and K. H. Burns, 2019 Transposable elements in human genetic disease. Nat. Rev. Genet. 20: 760-772. https://doi.org/10.1038/s41576-019-0165-8

Plasterk, R. H., Z. Izsvák, and Z. Ivics, 1999 Resident aliens: the Tc1/mariner superfamily of transposable elements. Trends Genet. 15: 326-332. https://doi.org/10.1016/s01689525(99)01777-1

Quinlan, A. R., and I. M. Hall, 2010 BEDTools: a flexible suite of utilities for comparing genomic features. Bioinformatics 26: 841-842. https://doi.org/10.1093/bioinformatics/btq033

R Core Team, 2014. R: A language and environment for statistical computing. R Foundation for Statistical Computing, Vienna, Austria. URL http://www.R-project.org/.

Rebollo, R., S. Farivar, and D. L. Mager, 2012 C-GATE - catalogue of genes affected by Transposable elements. Mobile DNA 3: 9. https://doi.org/10.1186/1759-8753-3-9

Reed, K. J., J. M. Svendsen, K. C. Brown, B. E. Montgomery, T. N. Marks et al., 2020 Widespread roles for piRNAs and WAGO-class siRNAs in shaping the germline transcriptome of Caenorhabditis elegans. Nucl. Acids Res. 48: 1811-1827. https://doi.org/10.1093/nar/gkz1178

Riddle, D. L., T. Blumenthal, B. J. Meyer, and J. R. Priess, editors, 1997 C. elegans II. 2nd edition. Cold Spring Harbor (NY): Cold Spring Harbor Laboratory Press; 1997. Section 
II, Origins of the Model. Available from:

https://www.ncbi.nlm.nih.gov/books/NBK20127/

Rizzon, C., G. Marais, M. Gouy, and C. Biémont, 2002 Recombination rate and the distribution of transposable elements in the Drosophila melanogaster genome. Genome Res. 12: 400-407. https://doi.org/10.1101/gr.210802

Robb, S. M. C., L. Lu, E. Valencia, J. M. Burnette 3rd, Y. Okumoto et al., 2013 The use of RelocaTE and unassembled short reads to produce high-resolution snapshots of transposable element generated diversity in rice. G3 (Bethesda) 3: 949-957. https://doi.org/10.1534/g3.112.005348

Rockman, M. V., and L. Kruglyak, 2009 Recombinational landscape and population genomics of Caenorhabditis elegans. PLoS Genet. 5: e1000419. https://doi.org/10.1371/journal.pgen.1000419

Rosenzweig, B., L. W. Liao, and D. Hirsh, 1983a Sequence of the C. elegans transposable element Tc1. Nucleic Acids Res. 11: 4201-4209. https://doi.org/10.1093/nar/11.12.4201

Rosenzweig, B., L. W. Liao, and D. Hirsh, 1983b. Target sequences for the C. elegans transposable element Tc1. Nucleic Acids Res. 11: 7137-7140. https://doi.org/10.1093/nar/11.20.7137

Shook, D. R., and T. E. Johnson, 1999 Quantitative trait loci affecting survival and fertilityrelated traits in Caenorhabditis elegans show genotype-environment interactions, pleiotropy and epistasis. Genetics 153: 1233-1243. https://doi.org/10.1093/genetics/153.3.1233

Sijen, T., and R. H. Plasterk, 2003 Transposon silencing in the Caenorhabditis elegans germ line by natural RNAi. Nature 426: 310-314. https://doi.org/10.1038/nature02107

Sivasundar, A., and J. Hey, 2003 Population genetics of Caenorhabditis elegans: the paradox of low polymorphism in a widespread species. Genetics 163:147-157. https://doi.org/10.1093/genetics/163.1.147

Sokal, R. R., and F. J. Rohlf, 1995 Biometry: The Principles and Practices of Statistic in Biological Research. W. H. Freeman and Company, New York.

Sulston, J. E., and S. Brenner, 1974 The DNA of Caenorhabditis elegans. Genetics 77: 95-104. https://doi.org/10.1093/genetics/77.1.95

Sultana, T., A. Zamborlini, G. Cristofari, and P. Lesage, 2017 Integration site selection by 
retroviruses and transposable elements in eukaryotes. Nat. Rev. Genet. 18: 292-308. https://doi.org/10.1038/nrg.2017.7

Thomas, C. G., W. Wang, R. Jovelin, R. Ghosh, T. Lomasko et al., 2015 Full-genome evolutionary histories of selfing, splitting, and selection in Caenorhabditis. Genome Res. 25: 667-678. https://doi.org/10.1101/gr.187237.114

van't Hof, A. E., N. Edmonds, M. Dalíková, F. Marec, and I. J. Saccheri, 2011 Industrial melanism in British peppered moths has a singular and recent mutational origin. Science 20: 958-960. https://doi.org/10.1126/science.1203043

Vaser, R., S. Adusumalli, S. N. Leng, M. Sikic, and P. C. Ng, 2016 SIFT missense predictions for genomes. Nat. Protoc. 11: 1-9. https://doi.org/10.1073/10.1038/nprot.2015.123

Vastenhouw, N. L., S. E. J. Fischer, V. J. P. Robert, K. L. Thijssen, A. G. Fraser et al., 2003 A genome-wide screen identifies 27 genes involved in transposon silencing in C. elegans. Curr. Biol. 13: 1311-1316. https://doi.org/10.1016/s0960-9822(03)00539-6

Vendrell-Mir, P., F. Barteri, M. Merenciano, J. González, J. M. Casacuberta et al., 2019 A benchmark of transposon insertion detection tools using real data. Mob. DNA 10: 53. https://doi.org/10.1186/s13100-019-0197-9

Vertino, A., S. Ayyadevara, J. J. Thaden, and R. J. Shmookler Reis, 2011 A narrow quantitative trait locus in C. elegans coordinately affects longevity, thermotolerance, and resistance to paraquat. Front. Genet. 2: 63. https://doi.org/10.3389/fgene.2011.00063

Wallis, D. C., D. A. H. Nguyen, C. J. Uebel, and C. M. Phillips, 2019 Visualization and quantification of transposon activity in Caenorhabditis elegans RNAi pathway mutants. G3 (Bethesda) 9: 3825-3832. https://doi.org/10.1534/g3.119.400639

Weick, E.-M., and E. A. Miska, 2014 piRNAs: from biogenesis to function. Development 141: 3458-3471. https://doi.org/10.1242/dev.094037

Wells, J. N., and C. Feschotte, 2020 A field guide to eukaryotic transposable elements. Annu. Rev. Genet. 54: 539-561. https://doi.org/10.1146/annurev-genet-040620-022145

Wood, W. B., R. Hecht, S. Carr, R. Vanderslice, N. Wolf et al., 1980 Parental effects and phenotypic characterization of mutations that affect early development in Caenorhabditis elegans. Dev. Biol. 74: 446-469. https://doi.org/10.1016/0012-1606(80)90445-5

Yigit, E., P. J. Batista, Y. Bei, K. M. Pang, C. C. Chen et al., 2006 Analysis of the C. elegans Argonaute family reveals that distinct argonautes act sequentially during RNAi. Cell 
127: 747-757. https://doi.org/10.1016/j.cell.2006.09.033

Yu, T., X. Huang, S. Dou, X. Tang, S. Luo et al., 2021 A benchmark and an algorithm for detecting germline transposon insertions and measuring de novo transposon insertion frequencies. Nucleic Acids Res. 49: e44. https://doi.org/10.1093/nar/gkab010

Yuhang, Z., T. C. Cheng, G. Huang, Q. Lu, M. D. Surleac et al., 2019 Transposon molecular domestication and the evolution of the RAG recombinase. Nature 569: 79-84. https://doi.org/10.1038/s41586-019-1093-7 
Table 1. Estimated number of Tc1 transposable elements in the reference $\mathrm{N} 2$ and three Bergerac strains of $\boldsymbol{C}$. elegans. The haploid number of Tc 1 as determined by several TE element callers in the McClintock pipeline (Nelson et al. 2017). The range, means and medians are calculated from estimates provided by six selected callers in the McClintock pipeline. Copy-number estimates of Tcl from ddPCR are included for comparison.

\begin{tabular}{c|cccccccccccc}
\hline \multicolumn{10}{c}{ Estimates of genomic Tc1 copy-number by McClintock } & & & \\
\hline $\begin{array}{c}\text { C. elegans } \\
\text { strain }\end{array}$ & ngs_te_mapper 2 & RelocaTE & TEMP2 & RetroSeq & TEFLoN & $\begin{array}{c}\text { Normalized } \\
\text { Tc1 Coverage }\end{array}$ & Range & Mean & Median & ddPCR \\
\hline N2 & 28 & 28 & 26 & - & 30 & 30 & $26-30$ & 28 & 28 & 29 \\
CB4851 & 448 & 455 & 463 & 435 & 444 & 546 & $435-546$ & 465 & 452 & 455 \\
$R$ W6999 & 582 & 551 & 601 & 580 & 578 & 668 & $551-668$ & 593 & 581 & 610 \\
$R$ W7000 & 741 & 755 & 759 & 741 & 723 & 836 & $723-836$ & 759 & 748 & 882 \\
\hline
\end{tabular}

a The RetroSeq caller of the McClintock program does not call non-reference TEs, hence an estimate was omitted for the N2 strain. 


\section{Table 2. Fitness of three $C$. elegans Bergerac strain relative to control worms of the} laboratory strain, $\mathbf{N 2}$. For the $\mathrm{N} 2$ control, 15 lines each with five replicates were established (maximum $n=75$ ). For each of the three Bergerac strains (CB4851, RW6999, and RW7000), we assayed 20 lines with five replicates each (maximum $n=100$ ). Estimates of the mean phenotype for four fitness-related traits are provided for each of the four strains.

\begin{tabular}{r|cccc}
\hline $\begin{array}{c}\text { C. elegans } \\
\text { strain }\end{array}$ & Productivity & $\begin{array}{c}\text { Survivorship to } \\
\text { Adulthood (\%) }\end{array}$ & $\begin{array}{c}\text { Longevity } \\
\text { (days) }\end{array}$ & $\begin{array}{c}\text { Developmental } \\
\text { Time (hours) }\end{array}$ \\
\hline N2 CONTROL & 309 & 98 & 14 & 48 \\
CB4851 & 75 & 91 & 10 & 60 \\
RW6999 & 107 & 89 & 9 & 53 \\
RW7000 & 43 & 83 & 10 & 60 \\
\hline
\end{tabular}


Table 3. Two-level nested ANOVA for productivity, survivorship to adulthood, longevity, and developmental time of N2 control and Bergerac strains CB4851, RW6999, and RW7000.

\begin{tabular}{|c|c|c|c|c|c|}
\hline Source of variation & $d f$ & SS & MS & $F_{s}$ & $F_{s}^{\prime}$ \\
\hline \multicolumn{6}{|l|}{ Productivity } \\
\hline Among groups & 3 & 3069207 & 1023069 & 403.83 & $309.93 * * * *$ \\
\hline Among lines & 69 & 217546 & 3153 & 1.25 & \\
\hline Within lines (error) & $\underline{224}$ & 567480 & 2533 & & \\
\hline Total & 296 & & & & \\
\hline \multicolumn{6}{|l|}{ Survivorship to Adulthood } \\
\hline Among groups & 3 & 0.945 & 0.315 & 23.88 & $16.80 * * * *$ \\
\hline Among lines & 69 & 1.289 & 0.019 & $1.42 *$ & \\
\hline Within lines (error) & $\underline{274}$ & 3.615 & 0.019 & & \\
\hline Total & 346 & & & & \\
\hline \multicolumn{6}{|l|}{ Longevity } \\
\hline Among groups & 3 & 1044 & 348.0 & 12.77 & $12.49 * * * *$ \\
\hline Among lines & 68 & 2029 & 29.8 & 1.10 & \\
\hline Within lines (error) & $\underline{220}$ & 5995 & 27.3 & & \\
\hline Total & 291 & & & & \\
\hline \multicolumn{6}{|l|}{ Developmental Time } \\
\hline Among groups & 3 & 7804 & 2601.3 & 35.51 & $34.06^{* * * *}$ \\
\hline Among lines & 69 & 5348 & 77.5 & 1.06 & \\
\hline Within lines (error) & $\underline{223}$ & 16337 & 73.3 & & \\
\hline Total & 295 & & & & \\
\hline
\end{tabular}

* significance level of 0.05

** significance level of 0.01

*** significance level of 0.001

$* * * *$ significance level of 0.0001 
Table 4. Motility- and size-associated traits of three $C$. elegans Bergerac strain relative to control worms of the laboratory strain, $\mathbf{N 2}$. Traits measures for each of the four strains are for $n=100$ worms across 10 plates ( 10 worms/plate). Mean values for all four motility-related traits are provided for each of the four strains.

\begin{tabular}{r|cccc}
\hline \multicolumn{1}{l}{$\begin{array}{l}\text { C. elegans } \\
\text { strain }\end{array}$} & $\begin{array}{c}\text { Speed } \\
(\mu \mathrm{m} / \mathrm{s})\end{array}$ & $\begin{array}{c}\text { Length } \\
(\mu \mathrm{m})\end{array}$ & $\begin{array}{c}\text { Area } \\
\left(\mu \mathrm{m}^{2}\right)\end{array}$ & $\begin{array}{c}\text { Direction Change } \\
(\text { radians/s) }\end{array}$ \\
\hline N2 control & 178 & 799 & 61,078 & 0.53 \\
CB4851 & 166 & 647 & 50,758 & 0.63 \\
$R W 6999$ & 121 & 675 & 49,966 & 0.59 \\
$R W 7000$ & 53 & 636 & 42,266 & 1.13 \\
\hline
\end{tabular}


Table 5. ANOVA analyses for four motility and size-associated traits in wild type N2 and three Bergerac strains (CB4851, RW6999, and RW7000) of C. elegans.

\begin{tabular}{|c|c|c|c|c|}
\hline Source of variation & $d f$ & SS & MS & $\boldsymbol{F}_{s}$ \\
\hline \multicolumn{5}{|l|}{ Speed $(\mu \mathrm{m} / \mathrm{s})$} \\
\hline Among groups & 3 & 96109 & 32036 & $37.57 * * *$ \\
\hline Within lines (error) & $\underline{36}$ & $\underline{30697}$ & 852 & \\
\hline Total & 39 & 126807 & & \\
\hline \multicolumn{5}{|l|}{ Length $(\mu \mathrm{m})$} \\
\hline Among groups & 3 & 169232 & 56410 & $22.58 * * * *$ \\
\hline Within lines (error) & $\underline{36}$ & $\underline{89950}$ & 2498 & \\
\hline Total & 39 & 259182 & & \\
\hline \multicolumn{5}{|l|}{$\operatorname{Area}\left(\mu \mathrm{m}^{2}\right)$} \\
\hline Among groups & 3 & 1789912485 & 596637495 & $19.59 * * * *$ \\
\hline Within lines (error) & $\underline{36}$ & $\underline{1096184461}$ & 30449568 & \\
\hline Total & 39 & 2886096946 & & \\
\hline \multicolumn{5}{|l|}{ Direction Change (radians/s) } \\
\hline Among groups & 3 & 2.288 & 0.763 & $28.15 * * * *$ \\
\hline Within lines (error) & $\underline{36}$ & $\underline{0.976}$ & 0.027 & \\
\hline Total & 39 & 3.264 & & \\
\hline
\end{tabular}

* significance level of 0.05

** significance level of 0.01

*** significance level of 0.001

$* * * *$ significance level of 0.0001 


\section{FIGURE LEGENDS}

Figure 1 Proliferation of the Tc 1 transposon in the Bergerac strains of C. elegans. (A) Counts of the Tc1 transposon in our laboratory isolate of Bristol N2 and three Bergerac strains, CB4851, RW6999, and RW7000 using various computational and molecular methods. Computational methods included were ngs_te_mapper, RelocaTE, TEMP2, RetroSeq, TEFLoN, and coverage. Copy-number estimation via ddPCR was the sole molecular method. (B) Counts of other/nonTc1 transposable elements are displayed for N2, CB4851, RW6999, and RW7000. Tc2 was the only transposable element with a marked increase in the three Bergerac lines compared to N2, while Tc4 and Tc5 were found to be marginally higher in CB4851.

Figure 2 Comparison of Tcl insertions predicted by McClintock component methods. The positional agreement of the McClintock component methods was assessed by assigning Tc 1 calls to windows (1,000 bp and 100,000 bp windows for non-reference and reference calls, respectively) followed by a comparison of the calls made by each component method. The Venn diagrams compare the agreement of Tc1 calls for (A) N2, (B) CB4851, (C) RW6999, and (D) RW7000. Computational methods included were ngs_te_mapper2 (blue), RelocaTE (red), TEMP2 (green), RetroSeq (yellow), and TEFLoN (brown). The vast majority of Tc 1 insertions were called by multiple methods.

Figure 3 Significant fitness reduction in Bergerac strains relative to N2. Means for four fitnessrelated traits, namely (A) productivity, (B) survivorship to adulthood, (C) longevity, and (D) developmental time observed in the N2 control, and the three Bergerac strains CB4851, RW6999, and RW7000. (E) Decline in mean relative fitness of the three Bergerac strains relative to the N2 strain. For simplicity, the mean relative fitness value for each of the four traits in the Bristol N2 control was scaled to a value of 1 (not displayed). For all panels, error bars represent one standard error. Significance was determined via ANOVA and is displayed as asterisks where $p \leq 0.05 * / 0.01 * * / 0.001 * * *$. Exact $p$-values for the pairwise strain comparisons using TukeyKramer HSD can be found in Supplemental Tables S2-S5. 
Figure 4 Physical size reduction and aberrant behavior of the Bergerac strains. Mean values for four traits measured in the motility analyses, namely (A) speed, (B) body length, (C) body area, (C), and direction change (D) in the N2 control, and the three Bergerac strains CB4851, RW6999, and RW7000. (E) Mean relative changes between the three Bergerac strains and the N2 control. For simplicity, the mean relative value for each of the four traits in the Bristol N2 control was scaled to a value of 1 (nor displayed). The stars on brackets summarize $p$-values for Tukey-Kramer HSD comparisons between Bergerac strains. Stars on top of bars reflect $p$-values for Tukey-Kramer HSD comparisons to N2. Exact $p$-values for the pairwise strain comparisons using Tukey-Kramer HSD can be found in Supplemental Tables S6-S9.

Figure 5 The Tcl insertion motif in the Bergerac strains. The vertical axis indicates the proportion of each base at four upstream and downstream positions from the insertion site. The motif for Tcl insertion sites was based on 1,659 insertions in this study and matched that presented in previous literature. Tcl invariably inserts between a $\mathrm{T}(-1)$ and an $\mathrm{A}(+1)$ with additional conserved $\mathrm{A} / \mathrm{T}$ bases at positions -4 and +4 .

Figure 6 The chromosomal distribution of Tcl elements in the Bergerac strains relative to N2. (A) Genomic map of Tcl insertions within each strain. Non-reference Tcl insertions are displayed in green, while reference Tc1 sites in Bristol N2 are shown in orange. (B) The proportion of Tc $l$ elements by chromosome. Tc $l$ elements were significantly overrepresented on the $\mathrm{X}$ chromosome in CB4851 $\left(\chi^{2}=51.87, p=5.92 \times 10^{-13}\right)$, and chromosomes $\mathrm{V}$ and $\mathrm{X}$ in $\operatorname{RW6999}\left(\chi^{2}=49.06, p=2.16 \times 10^{-9}\right)$ and $\operatorname{RW7000}\left(\chi^{2}=50.88, p=9.15 \times 10^{-10}\right)$.

Figure 7 The proportions of Tcl elements located in arms, cores and tips. Arms are associated with high recombination rates whereas cores and tips have low recombination rates. There were no significant differences in the distribution of Tc $l$ between these three domains $\left(\mathrm{N} 2: \chi^{2}=2.66\right.$, $p=0.21$; CB4851: $\chi^{2}=0.47, p=0.49$; RW6999: $\chi^{2}=4.39, p=0.11 ; \operatorname{RW7000}: \chi^{2}=4.81, p=$ $0.11)$.

Figure 8 The proportions of Tcl insertions across different chromatin domains. (A) Histone modification $\mathrm{H} 3 \mathrm{~K} 27 \mathrm{me} 3$ is associated with repressed chromatin and lowly expressed genes. All 
of the Tc1 positions in $\mathrm{N} 2$ were found in this domain. Tc1 insertions were more abundant in regions associated with $\mathrm{H} 3 \mathrm{~K} 27 \mathrm{me} 3$ than expected by chance in $\mathrm{CB} 4851\left(\chi^{2}=7.44, p=0.013\right)$ and RW6999 $\left(\chi^{2}=8.82, p=0.0089\right)$, but not RW7000 $\left(\chi^{2}=2.82, p=0.09\right)$. (B) Histone modifications $\mathrm{H} 3 \mathrm{~K} 9 \mathrm{mme} 1 / 2 / 3$ are associated with repetitive DNA. All of the Tc 1 positions in $\mathrm{N} 2$ were found in these domains. Tc 1 insertions were overrepresented in H3K9me1/2/3 domains in strains RW6999 $\left(\chi^{2}=5.06, p=0.049\right)$ and RW7000 $\left(\chi^{2}=7.06, p=0.024\right)$, but not in CB4851 $\left(\chi^{2}=7.06, p=0.44\right)$. (C) Histone modifications H3K4me1/2/3, H3K27ac, H4K8ac, H4K16ac are associated with promoters of highly expressed genes. In N2, Tcl insertions were entirely absent from these domains. Tc 1 insertions were underrepresented in domains associated with H3K4me1/2/3, H3K27ac, H4K8ac, H4K16ac and promoters of in all three Bergerac strains; CB4851 $\left(\chi^{2}=10.91, p=0.00287\right), \operatorname{RW6999}\left(\chi^{2}=5.96, p=0.0184\right)$; RW7000 $\left(\chi^{2}=\right.$ 6.79, $p=0.0184$ ). (D) Histone modifications H3K36me3 and H3K79me1/2/3 are associated with highly expressed genes. In N2, Tcl insertions were entirely absent from these domains. In all three Bergerac strains, Tc 1 insertions were underrepresented in these domains (CB4851: $\chi^{2}=$ 48.33, $p=3.60 \times 10^{-12} ; \mathrm{RW} 6999: \chi^{2}=75.28, p=8.17 \times 10^{-18} ; \mathrm{RW7000}: \chi^{2}=82.71, p=2.85 \times$ $\left.10^{-19}\right)$.

Figure 9 The proportion of Tc 1 elements in exons, introns, and intergenic regions of Bergerac strains in comparison to Bristol N2. In N2, the Tc1 insertions were only found in introns and intergenic regions. The proportion of $\mathrm{Tc} l$ insertions in exons, introns and intergenic regions was significantly different between Bristol N2 and the Bergerac strains $\left(G=54.26, p=6.54 \times 10^{-}\right.$ ${ }^{10}, d f=6$ ), with the Bergerac strains showing a far larger and fewer proportion of Tcl insertions in exonic regions and intronic regions, respectively. There was no significant difference among the three Bergerac strains in the proportion of $\mathrm{Tc} 1$ insertions in exons, introns, and intergenic regions $(G=4.28, p=0.37, d f=4)$. 
FIGURE 1

A

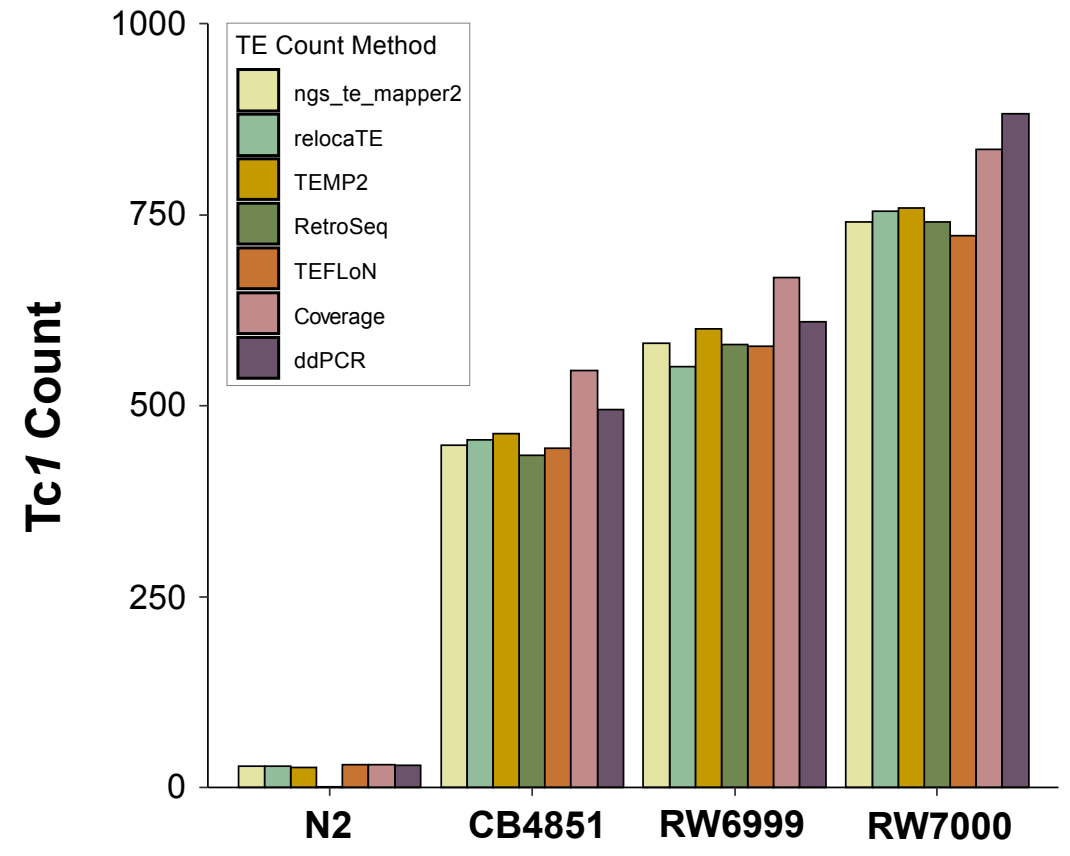

B
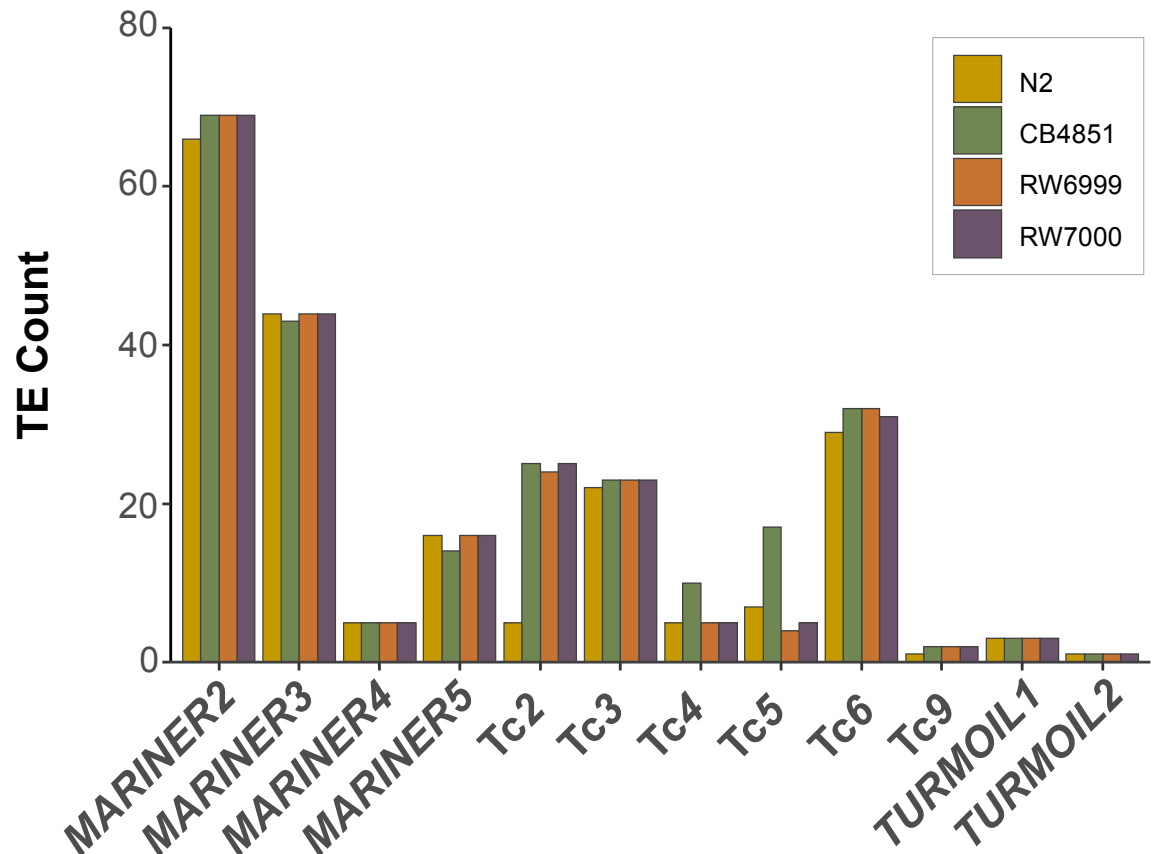
bioRxiv preprint doi: https://doi.org/10.1101/2022.02.02.478770; this version posted February 4, 2022. The copyright holder for this preprint (which was not certified by peer review) is the author/funder, who has granted bioRxiv a license to display the preprint in perpetuity. It is made available under aCC-BY 4.0 International license.

\section{FIGURE 2}

A

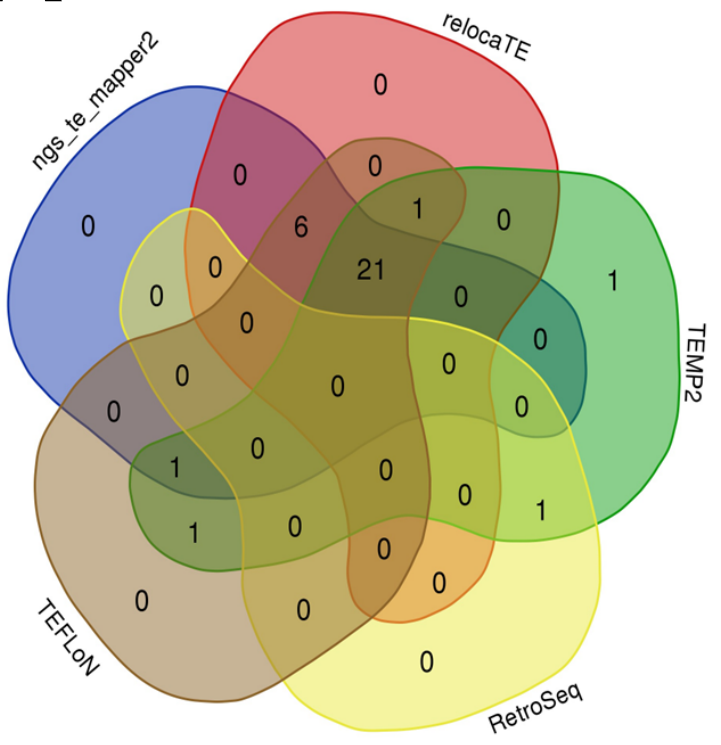

C

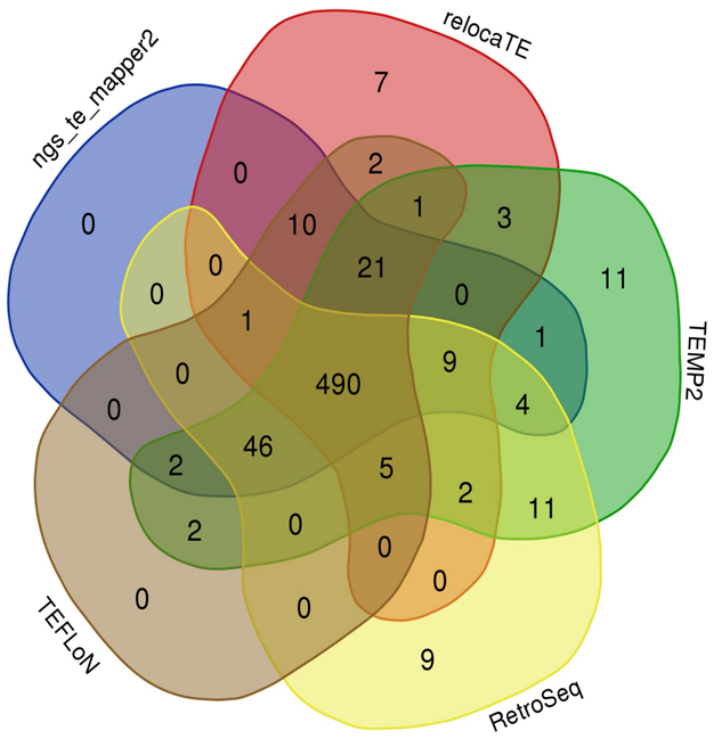

B

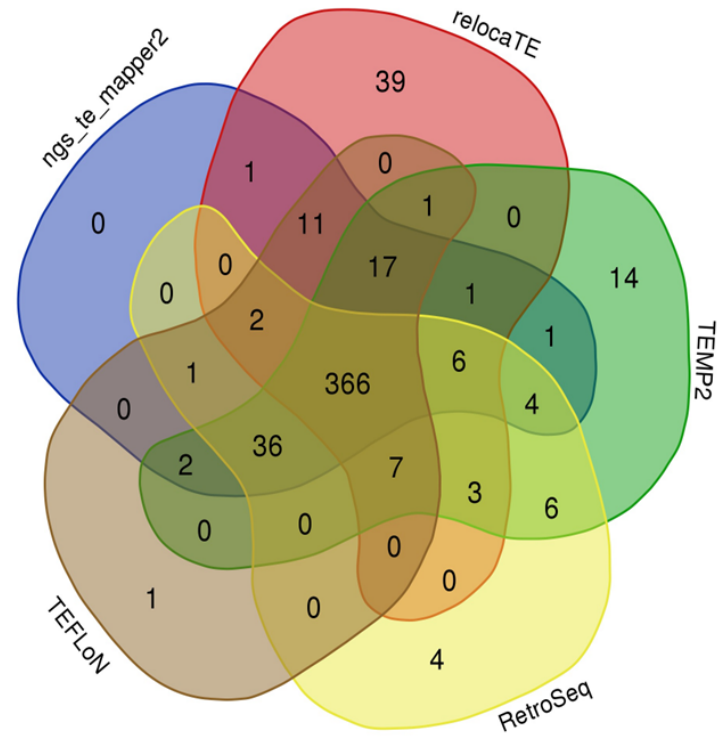

D

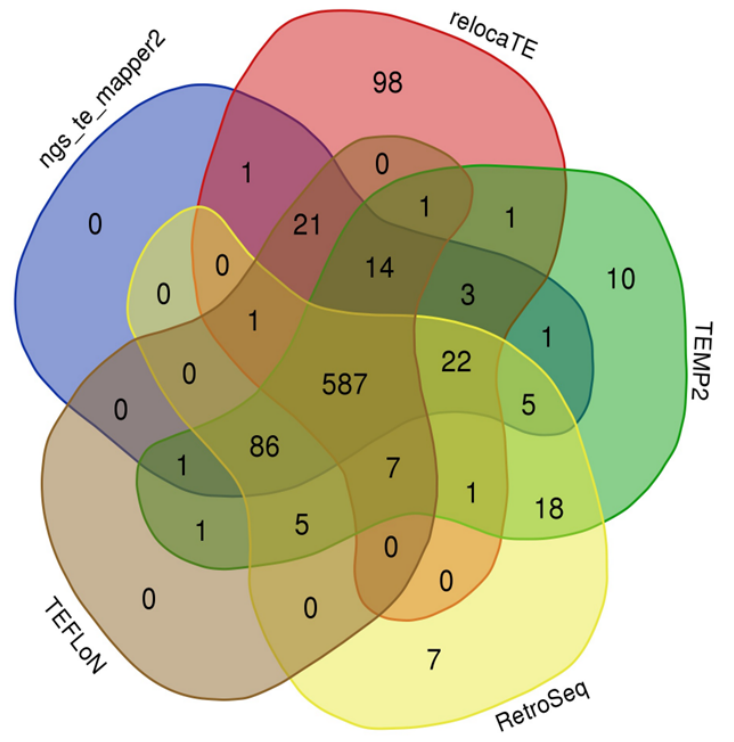




\section{FIGURE 3}

A

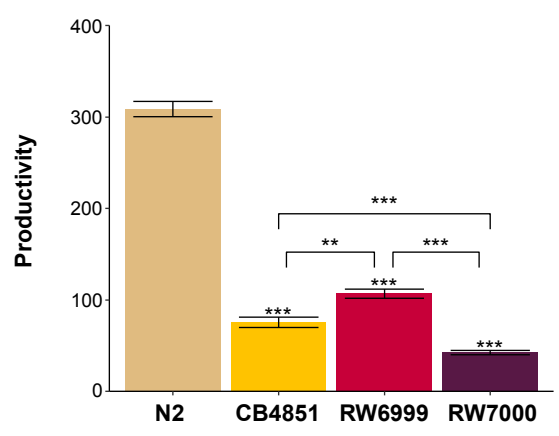

C

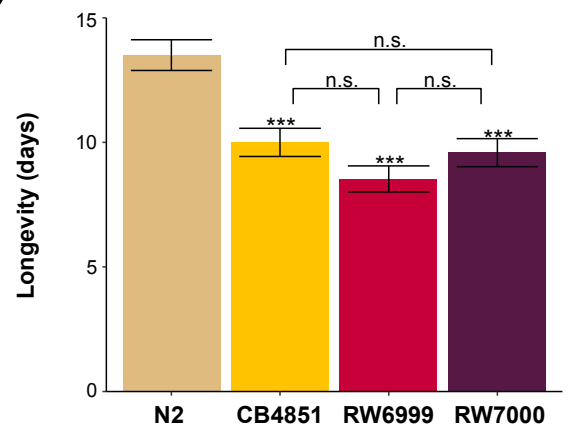

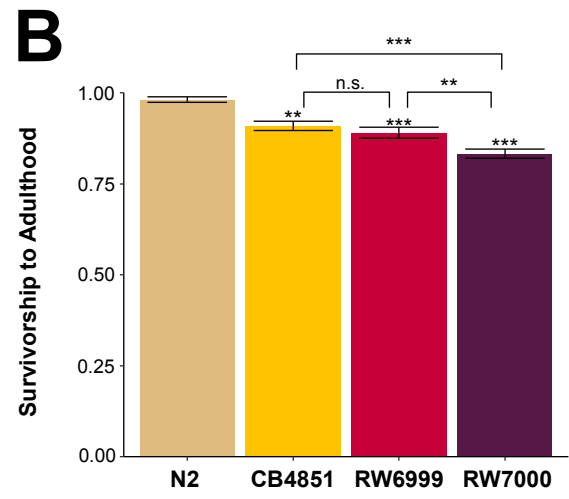

D

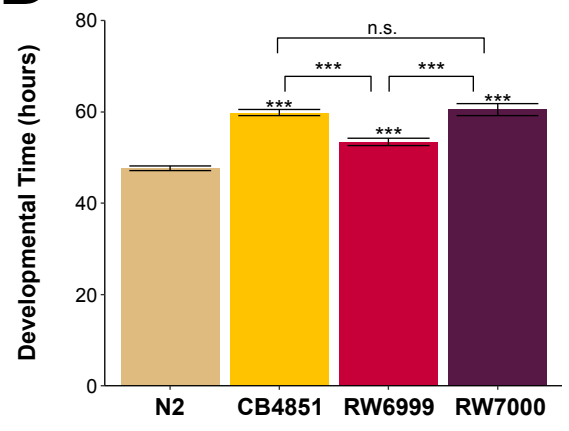

$\mathbf{E}$

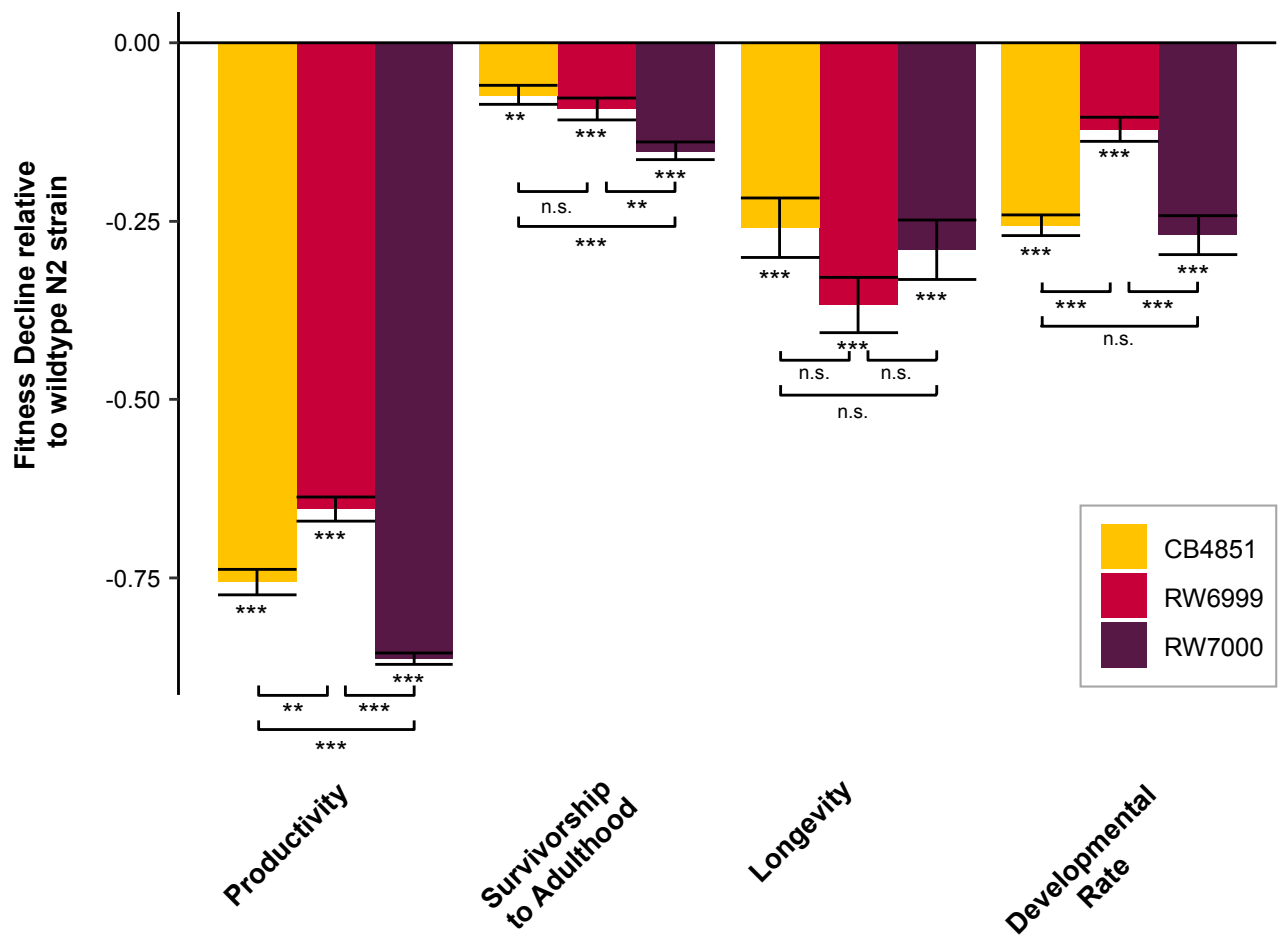


FIGURE 4

A

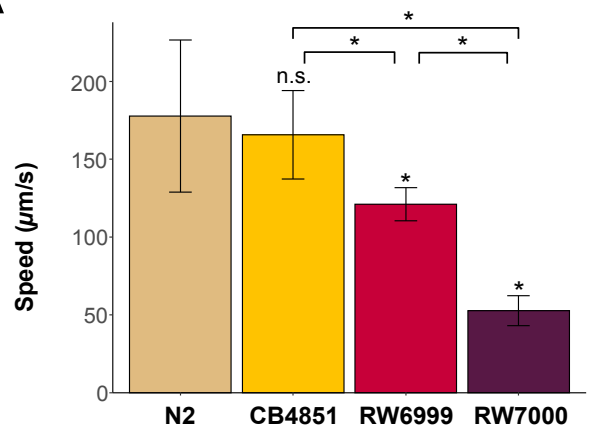

C

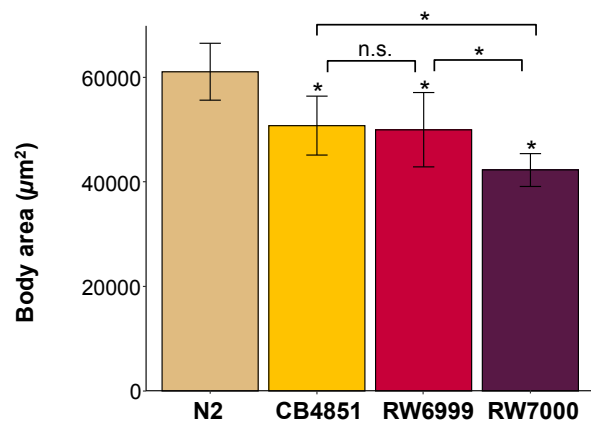

B

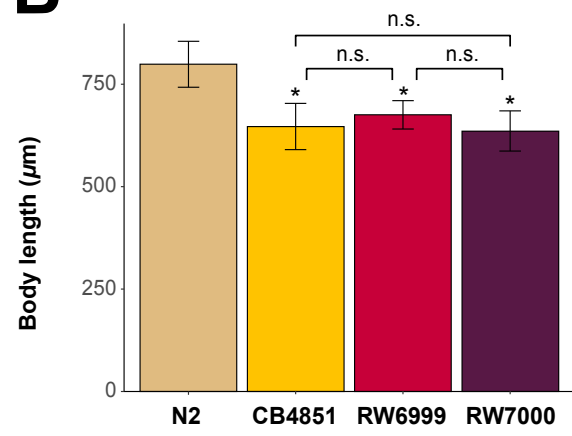

D

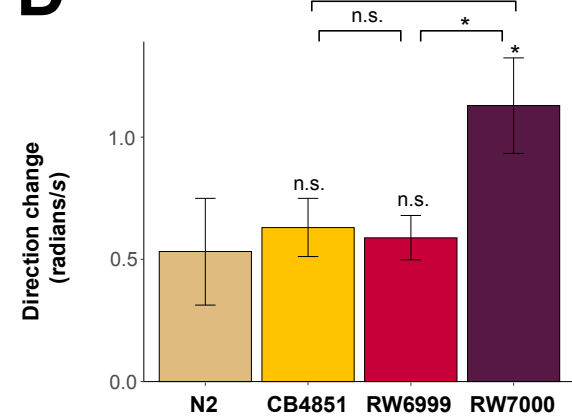

$\mathbf{E}$

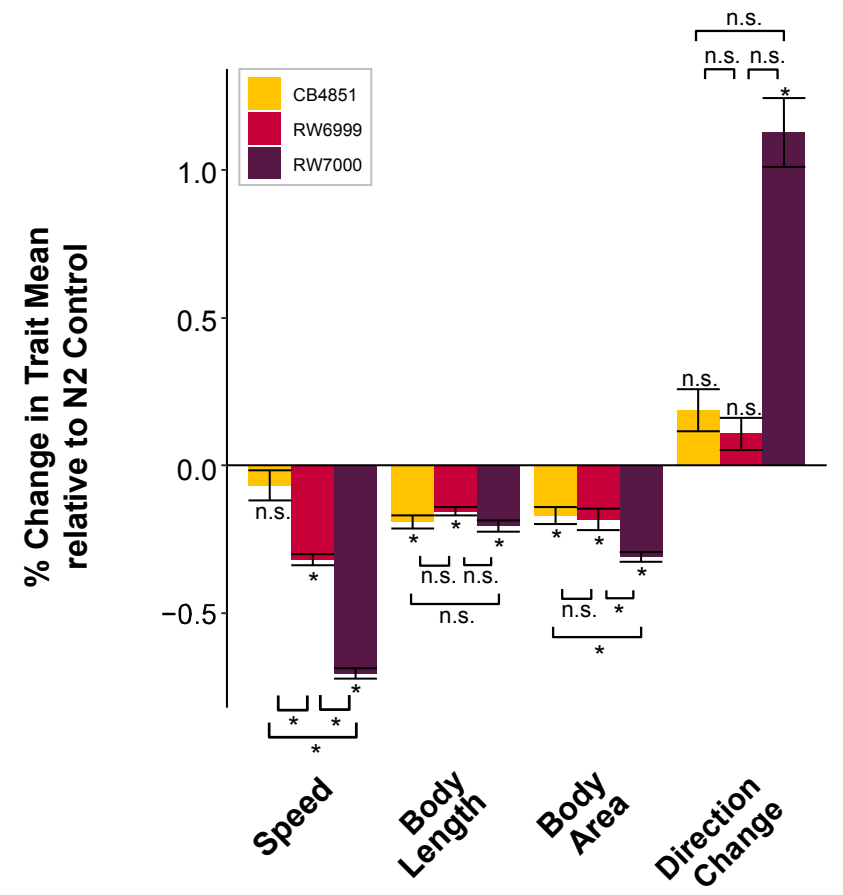


FIGURE 5

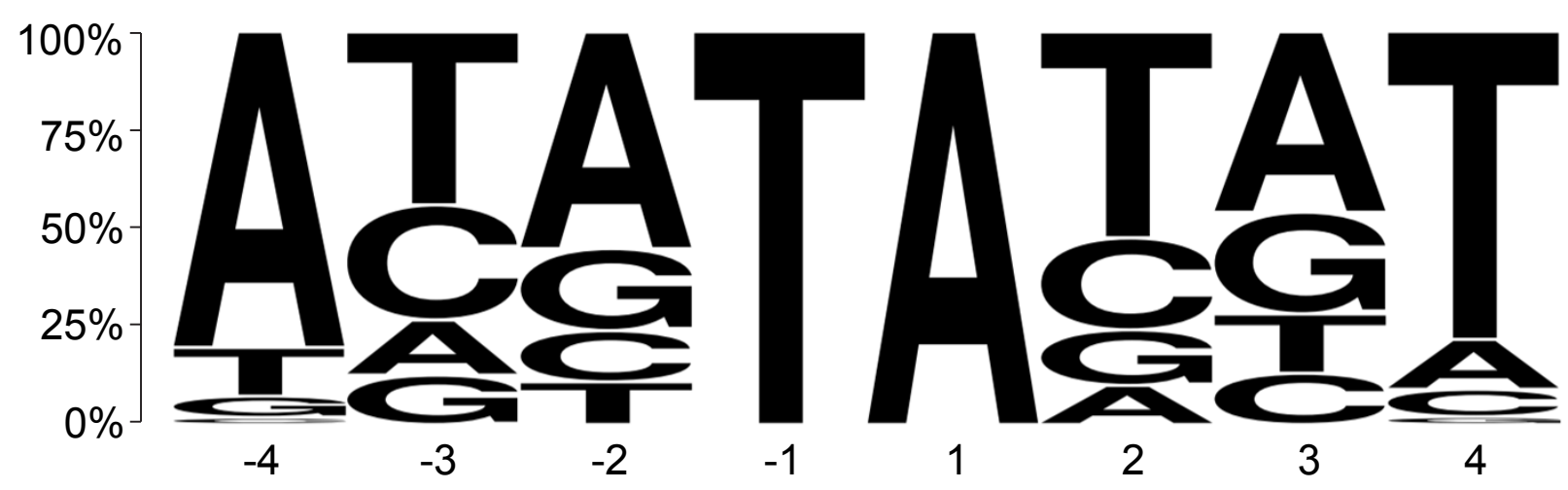


bioRxiv preprint doi: https://doi. org/10.1101/2022 02.02 .478770 . this version posted February 4 2022. The copyright holder for this preprint (which was not certified by peer review) is the author/funder, who has granted bioRxiv a license to display the preprint in perpetuity. It is made available under aCC-BY 4.0 International license.

\section{FIGURE 6}

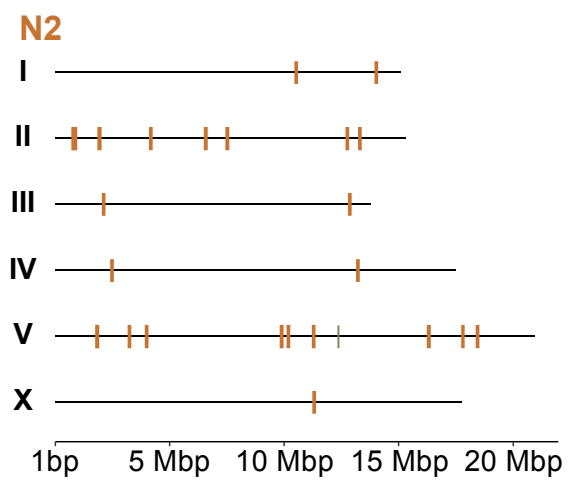

RW6999

I $-H-H H H W H H H H W H-H W H H-H H H$

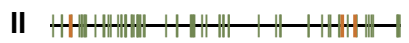

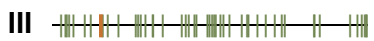

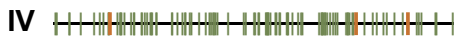

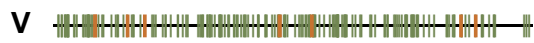

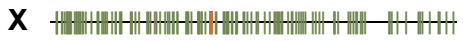

$1 \longdiv { \mathrm { bp } \quad 5 \mathrm { Mbp } 1 0 \mathrm { Mbp } 1 5 \mathrm { Mbp } 2 0 \mathrm { Mbp } }$

\section{CB4851}

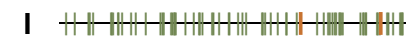

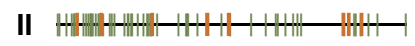

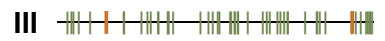

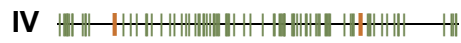

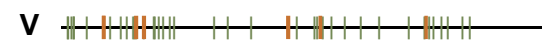

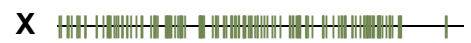

1bp $5 \mathrm{Mbp} 10 \mathrm{Mbp} 15 \mathrm{Mbp} 20 \mathrm{Mbp}$

RW7000

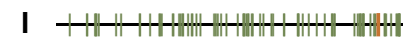

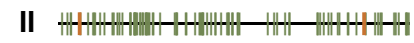

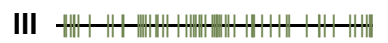

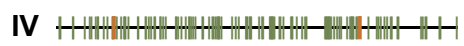

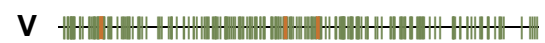

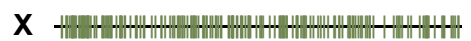

$1 \longdiv { \mathrm { bp } \quad 5 \mathrm { Mbp } 1 0 \mathrm { Mbp } 1 5 \mathrm { Mbp } 2 0 \mathrm { Mbp } }$

$0 \%$

$25 \%$

$50 \%$

$75 \%$

$100 \%$

N2

CB4851

RW6999

RW7000
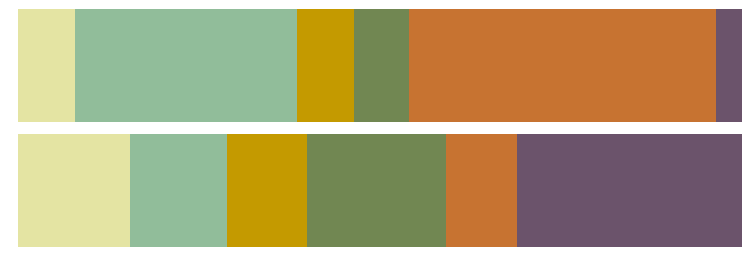

Chromosome

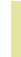
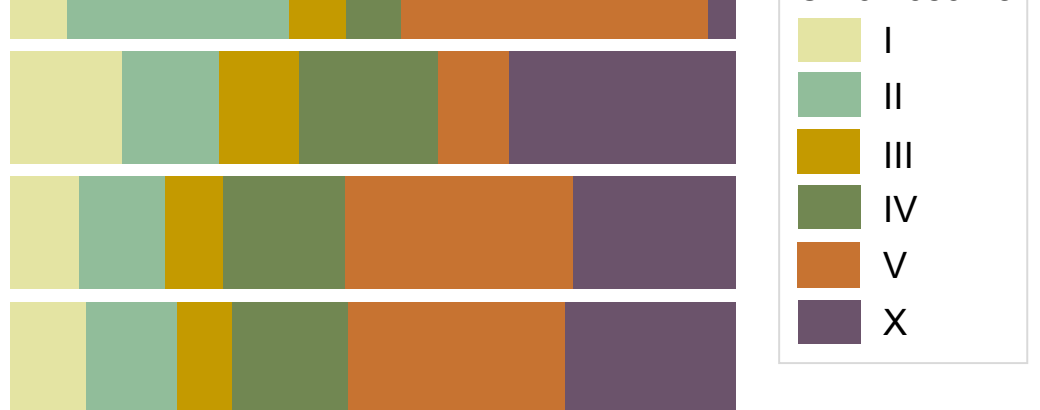
FIGURE 7

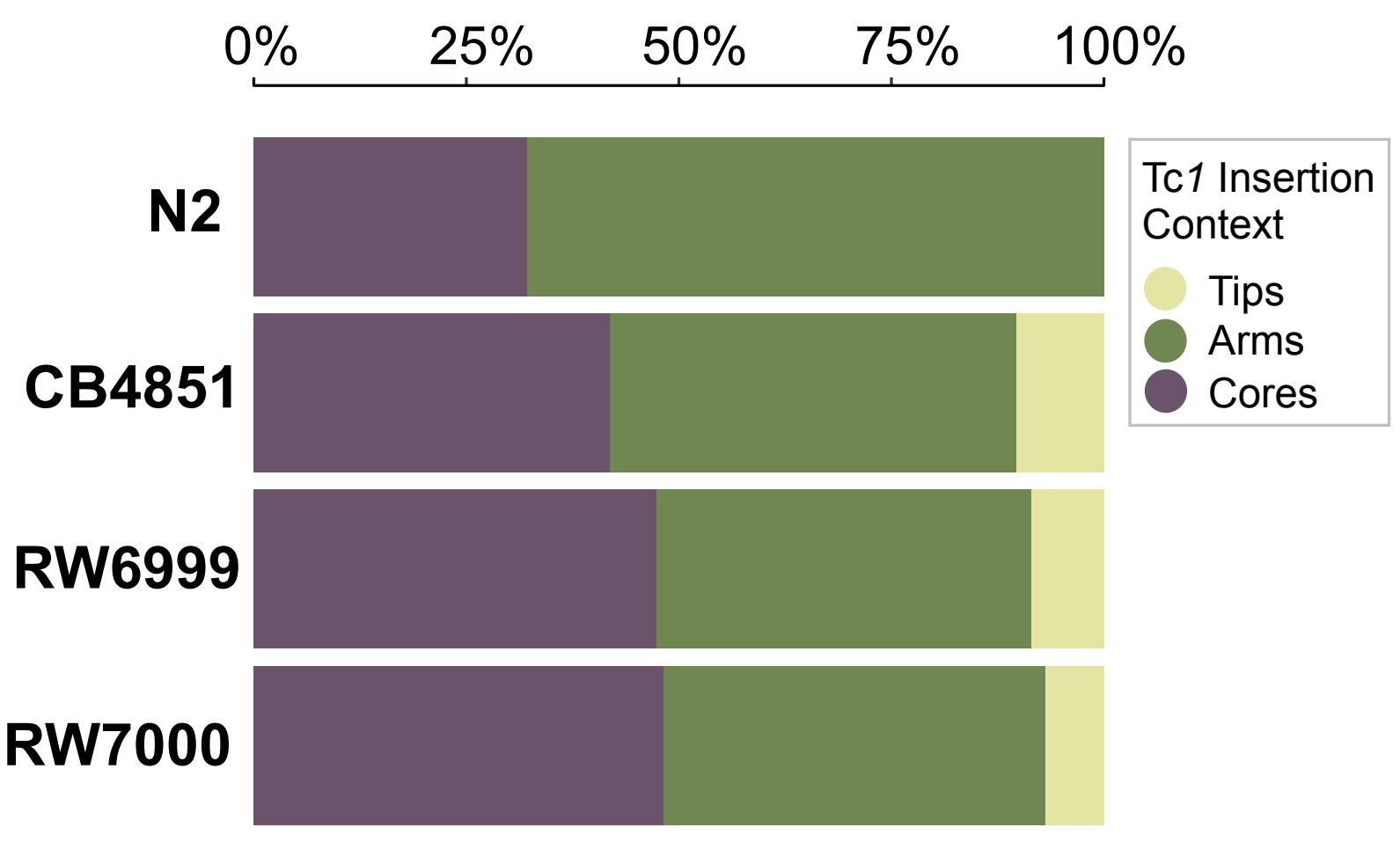




\section{FIGURE 8}

A

Lowly expressed genes

H3K27me3

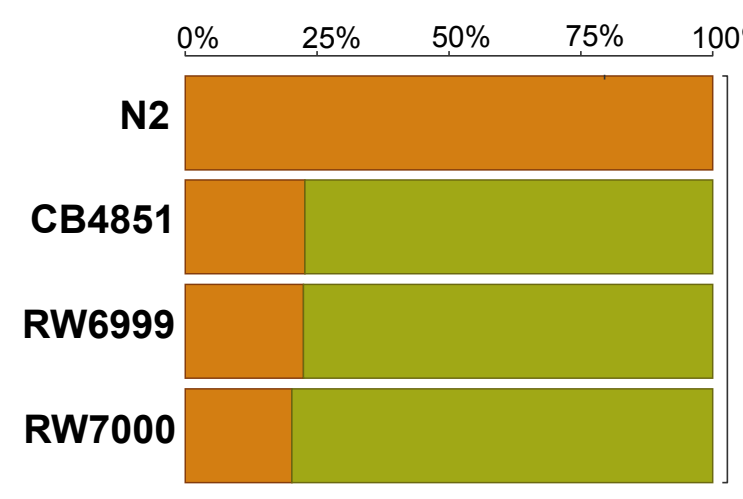

C

\section{Promoters of highly transcribed genes}

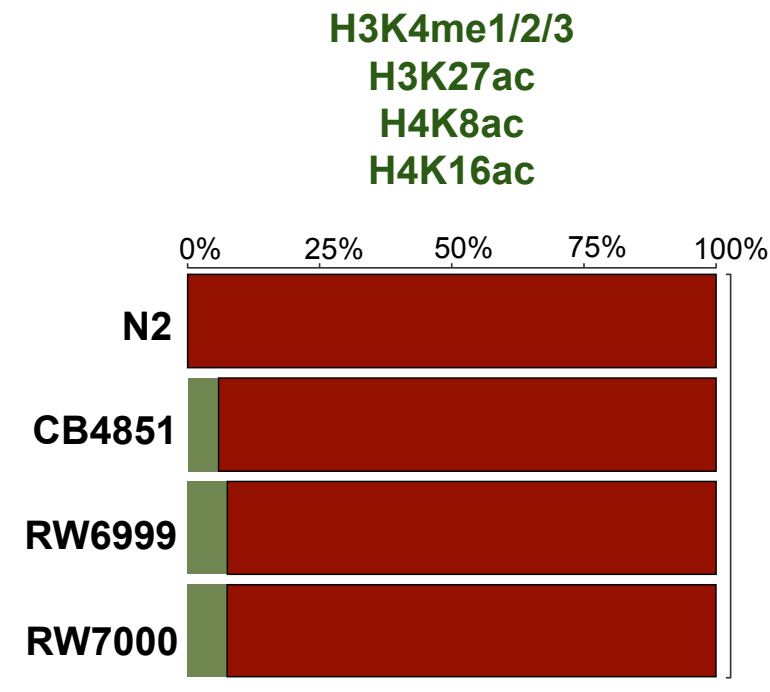

\section{Repetitive regions}

H3K9me1/2/3

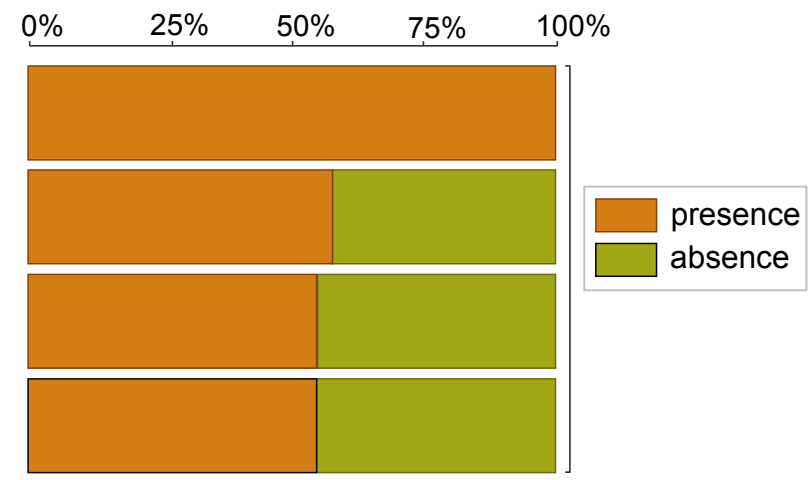

Highly expressed genes

H3K36me3

H3K79me1/2/3

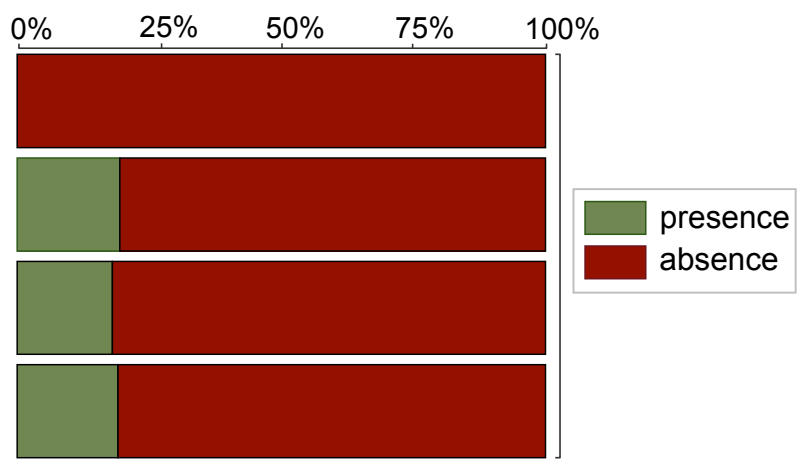




\section{FIGURE 9}

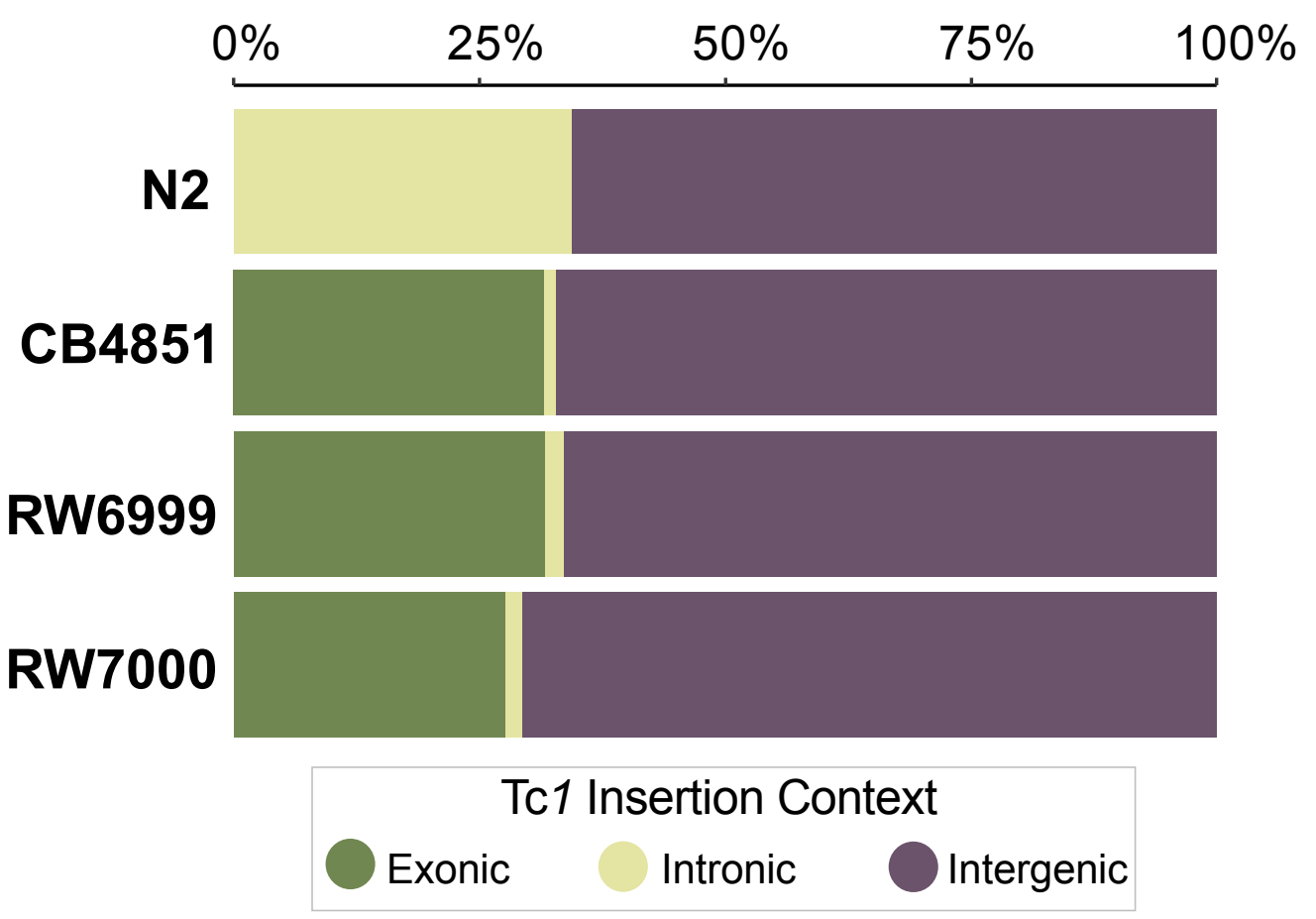

